\title{
Estimates of anisotropic Sobolev spaces with mixed norms for the Stokes system in a half-space
}

\author{
Tongkeun Chang and Kyungkeun Kang
}

\begin{abstract}
We are concerned with the non-stationary Stokes system with non-homogeneous external force and non-zero initial data in $\mathbb{R}_{+}^{n} \times(0, T)$. We obtain new estimates of solutions including pressure in terms of mixed anisotropic Sobolev spaces. As an application, some anisotropic Sobolev estimates are presented for weak solutions of the Navier-Stokes equations in a halfspace in dimension three.

2000 Mathematics Subject Classification. 35K51, 76D07.
\end{abstract}

Keywords and phrases: Stokes System, Navier-Stokes equations, anisotropic Sobolev space

\section{Introduction}

In this paper, we study the non-stationary Stokes system in a half-space $\mathbb{R}_{+}^{n} \times[0, T), n \geq 3$

$$
v_{t}-\Delta v+\nabla p=f, \quad \operatorname{div} v=0 \quad \text { in } Q_{T}^{+}:=\mathbb{R}_{+}^{n} \times[0, T),
$$

where $v: Q_{T}^{+} \rightarrow \mathbb{R}^{n}$ is the velocity field and $p: Q_{T}^{+} \rightarrow \mathbb{R}$ is the pressure. We consider the initial and boundary value problem of (1.1), whereby no slip boundary conditions are assigned, that is

$$
v(x, 0)=v_{0}(x) \quad \text { and } \quad v(x, t)=0, \quad x \in \partial \mathbb{R}_{+}^{n}=\mathbb{R}^{n-1} .
$$

The Stokes system is the linearized equations of the Navier-Stokes equations describing motions of incompressible and viscous fluid flows, which are given as follows:

$$
v_{t}-\Delta v+(v \cdot \nabla) v+\nabla p=f, \quad \operatorname{div} v=0 .
$$

Since Leray [19] proved existence of weak solutions of the Navier-Stokes equations (1.3), regularity of weak solutions has remained open in three dimensions (see also [12]). Lots of contributions have been made so far for uniqueness and regularity of the Navier-Stokes equations and many efforts to understand the Stokes system better have been also performed (see e.g. [22, 26], [18, [23, [6], [3], [8], 30, 4], [5, 11]). However, when boundaries of domains are not empty, comparatively small number of results have been known, because of difficulty of pressure up to the boundary (see e.g 24, [25], [16], 10]). Among results with non-empty boundaries, we recall the following $L^{p}$ estimates for Strokes system in half-space (see e.g. [27): Let $1<p<\infty$ and $f \in L^{p}\left(Q_{T}^{+}\right)$and $v_{0} \in \dot{B}^{2-\frac{2}{p}}\left(\mathbb{R}_{+}^{n}\right)$, where $\dot{B}^{2-\frac{2}{p}}\left(\mathbb{R}_{+}^{n}\right)$ is a homogeneous Besov space. Then the solution of the Stokes system (1.1) and (1.2) satisfies

$$
\left\|v_{t}\right\|_{L^{p}\left(Q_{T}^{+}\right)}+\left\|\nabla^{2} v\right\|_{L^{p}\left(Q_{T}^{+}\right)}+\|\nabla p\|_{L^{p}\left(Q_{T}^{+}\right)} \leq c\|f\|_{L^{p}\left(Q_{T}^{+}\right)}+c\left\|v_{0}\right\|_{\dot{B}_{p}^{2-\frac{2}{p}}\left(\mathbb{R}_{+}^{n}\right)} .
$$

In [7, using the theory of Stokes operator, $v$ satisfies the following estimate of fractional derivatives for $v_{0}=0$ :

$$
\int_{0}^{T}\left\|\left(\frac{d}{d t}\right)^{1-\alpha} v\right\|_{L^{p}\left(\mathbb{R}_{+}^{n}\right)}^{r} d t+\int_{0}^{T}\left\|A_{p}^{1-\alpha} v\right\|_{L^{p}\left(\mathbb{R}_{+}^{n}\right)}^{r} d t \leq c \int_{0}^{T}\left\|A_{p}^{-\alpha} f\right\|_{L^{p}\left(\mathbb{R}_{+}^{n}\right)}^{r} d t
$$


where $A_{p}$ is the Stokes operator and $0<\alpha<1$. As a consequence, when $f=\operatorname{div} F$ with $F=$ $\left(F_{i j}\right)_{i, j=1, \cdots, n}$ and $\alpha=1 / 2$, (1.5) yields the following a priori estimate:

$$
\int_{0}^{T}\left\|\left(\frac{d}{d t}\right)^{\frac{1}{2}} v\right\|_{L^{p}\left(\mathbb{R}_{+}^{n}\right)}^{r} d t+\int_{0}^{T}\|\nabla v\|_{L^{p}\left(\mathbb{R}_{+}^{n}\right)}^{r} d t \leq c \int_{0}^{T}\|F\|_{L^{p}\left(\mathbb{R}_{+}^{n}\right)}^{r} d t .
$$

Estimates for pressure were, however, not given in [7. Koch and Solonnikov [17] consider the Stokes system (1.1) with $f=\nabla \cdot F$, zero initial data and no-slip boundary conditions in three dimensional half space, and established that

$$
\|\nabla v\|_{L^{p}\left(\mathbb{R}_{+}^{3} \times(0, T)\right)} \leq c\|F\|_{L^{p}\left(\mathbb{R}_{+}^{3} \times(0, T)\right)} .
$$

Furthermore, it was also shown that there exists a $F \in L^{p}\left(\mathbb{R}_{+}^{3} \times(0, T)\right)$ such that corresponding pressure $p$ of (1.1) is not even in $L^{p}\left(\mathbb{R}_{+}^{3} \times(0, T)\right.$ ) (see [17, Theorem 1.3]). For comparison, such result is quite different to that of the entire space $\mathbb{R}^{3}$, whereby $(v, p)$ of Stokes system satisfies the following estimate:

$$
\|\nabla v\|_{L^{p}\left(\mathbb{R}^{3} \times(0, T)\right)}+\|p\|_{L^{p}\left(\mathbb{R}^{3} \times(0, T)\right)} \leq c\|F\|_{L^{p}\left(\mathbb{R}^{3} \times(0, T)\right)} .
$$

The main objective of this paper is to look for relevant function classes that $f$ and $v_{0}$ belong to such that control of pressure similar to (1.8) holds in a half-space (see Theorem 1.1 and Theorem 1.2). As an application of such estimate for the Stokes system, we present a new estimate especially including the pressure of the Navier-Stokes equations in a half space (see Theorem 1.3).

Before we state our main results, we remind some function classes, which are useful for our purpose. Let $\alpha \in \mathbb{R}^{n}$ and $1<p, q<\infty$. We mean by $H_{p}^{\alpha}\left(\mathbb{R}_{+}^{n}\right)$ a generalized Sobolev space in a half-space and we also write a homogeneous Sobolev space as $\dot{H}_{p}^{\alpha}\left(\mathbb{R}_{+}^{n}\right)$. Besides, we indicate that $B_{p q}^{\alpha}\left(\mathbb{R}_{+}^{n}\right)$ and $\dot{B}_{p q}^{\alpha}\left(\mathbb{R}_{+}^{n}\right)$ are usual Besov space and homogeneous Besov space, respectively. In addition, we define function spaces $H_{p, 0}^{\alpha}\left(\mathbb{R}_{+}^{n}\right), \dot{H}_{p, 0}^{\alpha}\left(\mathbb{R}_{+}^{n}\right), B_{p q, 0}^{\alpha}\left(\mathbb{R}_{+}^{n}\right)$ and $\dot{B}_{p q, 0}^{\alpha}\left(\mathbb{R}_{+}^{n}\right)$ as the closures of $C_{c}^{\infty}\left(\mathbb{R}_{+}^{n}\right)$ in $H_{p}^{\alpha}\left(\mathbb{R}_{+}^{n}\right), \dot{H}_{p}^{\alpha}\left(\mathbb{R}_{+}^{n}\right), B_{p q}^{\alpha}\left(\mathbb{R}_{+}^{n}\right)$ and $\dot{B}_{p q}^{\alpha}\left(\mathbb{R}_{+}^{n}\right)$. We denote some anisotropic Sobolev spaces by $H_{p q}^{\alpha, \frac{1}{2} \alpha}\left(\mathbb{R}_{+}^{n} \times(0, T)\right)$ and $H_{p q, 0}^{\alpha, \frac{1}{2} \alpha}\left(\mathbb{R}_{+}^{n} \times(0, T)\right)$ (see Section 2 for more details).

Now we are ready to state our first main result.

Theorem 1.1. Let $0 \leq \alpha \leq 2,0<T \leq \infty$ and $1<p, q<\infty$. Suppose that $f \in H_{p q, 0}^{\alpha-2, \frac{1}{2} \alpha-1}\left(\mathbb{R}_{+}^{n} \times\right.$ $(0, T))$. and $v_{0} \in B_{p q, 0}^{\alpha-\frac{2}{q}}\left(\mathbb{R}_{+}^{n}\right)$ with $\operatorname{div} f=0$ and $\operatorname{div} v_{0}=0$ in the sense of distributions. Then, there exists a unique solution $v$ in $H_{p q}^{\alpha, \frac{1}{2} \alpha}\left(\mathbb{R}_{+}^{n} \times(0, T)\right)$ of (1.1) such that the following estimate is satisfied:

$$
\|v\|_{H_{p q}^{\alpha, \frac{1}{2} \alpha}{ }_{\left(\mathbb{R}_{+}^{n} \times(0, T)\right)}} \leq c\|f\|_{H_{p q, 0}^{\alpha-2, \frac{1}{2} \alpha-1}\left(\mathbb{R}_{+}^{n} \times(0, T)\right)}+c\left\|v_{0}\right\|_{B_{p q, 0}^{\alpha-\frac{2}{q}}\left(\mathbb{R}_{+}^{n}\right)} .
$$

Moreover, if $1+1 / p<\alpha \leq 2$, then $p \in L_{q}\left(0, T ; H_{p}^{\alpha-1}\left(\mathbb{R}_{+}^{n}\right)\right)$ such that

$$
\|p\|_{L_{q}\left(0, T ; H_{p}^{\alpha-1}\left(\mathbb{R}_{+}^{n}\right)\right)} \leq c\|f\|_{H_{p q, 0}^{\alpha-2, \frac{1}{2} \alpha-1}\left(\mathbb{R}_{+}^{n} \times(0, T)\right)}+c\left\|v_{0}\right\|_{B_{p q, 0}^{\alpha-\frac{2}{q}}\left(\mathbb{R}_{+}^{n}\right)} .
$$

If $f \in \dot{H}_{p q, 0}^{\alpha-2, \frac{1}{2} \alpha-1}\left(\mathbb{R}_{+}^{n} \times(0, T)\right)$ and $v_{0} \in \dot{B}_{p q, 0}^{\alpha-\frac{2}{q}}\left(\mathbb{R}_{+}^{n}\right)$, then $v \in \dot{H}_{p q}^{\alpha, \frac{1}{2} \alpha}\left(\mathbb{R}_{+}^{n} \times(0, T)\right)$ and satisfies

$$
\|v\|_{\dot{H}_{p q}^{\alpha, \frac{1}{2} \alpha}\left(\mathbb{R}_{+}^{n} \times(0, T)\right)} \leq c\|f\|_{\dot{H}_{p q, 0}^{\alpha-2, \frac{1}{2} \alpha-1}\left(\mathbb{R}_{+}^{n} \times(0, T)\right)}+c\left\|v_{0}\right\|_{\dot{B}_{p q, 0}^{\alpha-\frac{2}{q}}\left(\mathbb{R}_{+}^{n}\right)} .
$$

Furthermore, if $1+1 / p<\alpha \leq 2$, then $p \in L_{q}\left(0, T ; \dot{H}_{p}^{\alpha-1}\left(\mathbb{R}_{+}^{n}\right)\right)$ such that

$$
\|p\|_{L_{q}\left(0, T ; \dot{H}_{p}^{\alpha-1}\left(\mathbb{R}_{+}^{n}\right)\right)} \leq c\|f\|_{\dot{H}_{p q, 0}^{\alpha-2, \frac{1}{2} \alpha-1}\left(\mathbb{R}_{+}^{n} \times(0, T)\right)}+c\left\|v_{0}\right\|_{\dot{B}_{p q, 0}^{\alpha-\frac{2}{q}}\left(\mathbb{R}_{+}^{n}\right)} .
$$


Remark In Theorem 1.1, the solution $v$ means in the following distribution sense: For any smooth vector field $\psi \in \mathcal{C}_{0}^{\infty}\left(\mathbb{R}_{+}^{n} \times(0, T)\right)$ with $\operatorname{div} \psi=0$ and for any smooth scalar function $\phi \in \mathcal{C}_{0}^{\infty}\left(\mathbb{R}_{+}^{n} \times\right.$ $(0, T)) v$ satisfies

$$
\int_{0}^{T} \int_{\mathbb{R}_{+}^{n}} v \cdot\left(-\psi_{t}-\Delta \psi\right) d x d t=0, \quad \int_{0}^{T} \int_{\mathbb{R}_{+}^{n}} v \cdot \nabla \phi d x d t=0
$$

Remark The divergence free conditions for $f$ and $v_{0}$ in Theorem 1.1 means that $\langle f, \nabla \phi\rangle=0$ and $\left\langle v_{0}, \nabla \phi\right\rangle=0$ for any $\phi \in \mathcal{C}_{0}^{\infty}\left(\mathbb{R}^{n}\right)$. We also remark that the constant $c$ in (1.11)-(1.12) is independent of $T$ for the homogeneous case.

The following is a consequence of Theorem 1.1]

Theorem 1.2. Let $1<p, q<\infty$ and $0 \leq \beta \leq 1$. Suppose that $f=\nabla \cdot F$, where $F=\left(F_{i j}\right)_{i, j=1, \cdots, n}$ is a tensor such that $F \in L_{q}\left(0, T ; H_{p, 0}^{\beta}\left(\mathbb{R}_{+}^{n}\right)\right)$ and $v_{0} \in B_{p q, 0}^{1+\beta-\frac{2}{q}}\left(\mathbb{R}_{+}^{n}\right)$ with $\operatorname{div} f=0$ and $\operatorname{div} v_{0}=0$ in the sense of distributions. Then, there exists a unique solution $v \in H_{p q}^{1+\beta, \frac{1+\beta}{2}}\left(\mathbb{R}_{+}^{n} \times(0, T)\right)$ of (1.1) such that the following estimate is satisfied:

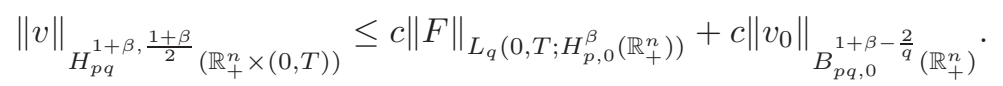

Furthermore, if $\beta>1 / p$, then $p \in L_{q}\left(0, T ; H_{p}^{\beta}\left(\mathbb{R}_{+}^{n}\right)\right)$ of (1.1) such that

$$
\|p\|_{L_{q}\left(0, T ; H_{p}^{\beta}\left(\mathbb{R}_{+}^{n}\right)\right)} \leq c\|F\|_{L_{q}\left(0, T ; H_{p, 0}^{\beta}\left(\mathbb{R}_{+}^{n}\right)\right)}+c\left\|v_{0}\right\|_{B_{p q, 0}^{1+\beta-\frac{2}{q}}\left(\mathbb{R}_{+}^{n}\right)} .
$$

If $F \in L_{q}\left(0, T ; \dot{H}_{p, 0}^{\beta}\left(\mathbb{R}_{+}^{n}\right)\right.$ and $v_{0} \in \dot{B}_{p q, 0}^{1+\beta-\frac{2}{q}}\left(\mathbb{R}_{+}^{n}\right)$, there exist $v \in \dot{H}_{p q}^{1+\beta, \frac{1+\beta}{2}}\left(\mathbb{R}_{+}^{n} \times(0, T)\right)$ with $\beta \geq 0$ and $p \in L_{q}\left(0, T ; \dot{H}_{p}^{\beta}\left(\mathbb{R}_{+}^{n}\right)\right)$ with $\beta>1 / p$ such that the estimates (1.14) and (1.15) are replaced by

$$
\begin{gathered}
\|v\|_{\dot{H}_{p q}^{1+\beta, \frac{1+\beta}{2}}}\left(\mathbb{R}_{+}^{n} \times(0, T)\right) \\
\|p\|_{L_{q}\left(0, T ; \dot{H}_{p}^{\beta}\left(\mathbb{R}_{+}^{n}\right)\right)} \leq c\|F\|_{L_{q}\left(0, T ; \dot{H}_{p, 0}^{\beta}\left(\mathbb{R}_{+}^{n}\right)\right)}+c\left\|v_{0}\right\|_{\dot{B}_{p q, 0}^{1+\beta-\frac{2}{q}}\left(\mathbb{R}_{+}^{n}\right)} . \\
\end{gathered}
$$

Although the proof of Theorem 1.2 is rather straightforward, the details will be, for clarity, presented in section 5 .

Remark In Theorem 1.2, in case that $p<n / \beta$, we observe due to Sobolev imbedding that

$$
\|p\|_{L_{q}\left(0, T ; L_{\tilde{p}}\left(\mathbb{R}_{+}^{n}\right)\right)} \leq c\|F\|_{L_{q}\left(0, T ; H_{p, 0}^{\beta}\left(\mathbb{R}_{+}^{n}\right)\right)}+c\left\|v_{0}\right\|_{B_{p q, 0}^{1+\beta-\frac{2}{q}}\left(\mathbb{R}_{+}^{n}\right)},
$$

where $1 / \tilde{p}=1 / p-\beta / n$. Compared the result in [17 to ours, $F$ was assumed only in $L_{x, t}^{p}\left((0, T) \times \mathbb{R}_{+}^{n}\right)$ in [17, which allows an example that the pressure is not even in $L_{x, t}^{p}\left((0, T) \times \mathbb{R}_{+}^{n}\right)$. In contrast, Theorem 1.2 shows that if $F$ has a bit better regularity in spatial variables, the pressure $p$ can be found in $L_{x, t}^{p}\left((0, T) \times \mathbb{R}_{+}^{n}\right)$ with suitable choice of $q$ and $\tilde{p}$ in (1.18).

As an application of the estimates of Stokes system, we consider the incompressible Navier-Stokes equations:

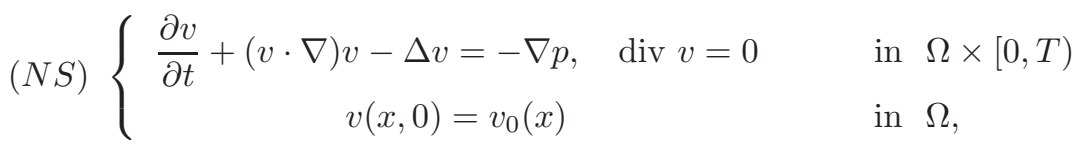


where $v$ and $p$ are the flow velocity and the scalar pressure, respectively. The initial data satisfy the compatibility condition, i.e., $\operatorname{div} v_{0}=0$ and no slip boundary condition is imposed for velocity $v$ at the boundary $\partial \Omega$, namely

$$
v(x, t)=0, \quad x \in \partial \Omega .
$$

In case that $\Omega=\mathbb{R}^{3}$, the following a priori estimate of Calderón-Zygmund type is well-known:

$$
\|p\|_{L^{q}\left(\mathbb{R}^{3}\right)} \leq C\|v\|_{L^{2 q}\left(\mathbb{R}^{3}\right)}^{2}, \quad 1<q<\infty .
$$

Therefore, when $\Omega=\mathbb{R}^{3}$, it is straightforward that weak solutions of (1.19) satisfy

$$
\|p\|_{L^{q}\left(\mathbb{R}^{3} \times I\right)} \leq C\|v\|_{L^{2 q}\left(\mathbb{R}^{3} \times I\right)}^{2}, \quad 1<q \leq \frac{5}{3} .
$$

Weak solutions are defined in section 5 (see Definition 5.1). However, it is not clear whether or not (1.21)-(1.22) is valid for the case that $\Omega$ has non-empty boundaries with no-slip condition (1.20). Instead, the following estimate is known for the gradient of pressure (see e.g. [27] and [9]):

$$
\begin{aligned}
& \|\nabla p\|_{L^{m}\left(0, T ; L^{l}\left(\mathbb{R}_{+}^{3}\right)\right)} \leq C\|v \nabla v\|_{L^{m}\left(0, T ; L^{l}\left(\mathbb{R}_{+}^{3}\right)\right)}+C\left\|v_{0}\right\|_{B_{p q, 0}^{1-\frac{2}{q}}\left(\mathbb{R}_{+}^{3}\right)} \\
& \leq C=C\left(\|u\|_{L^{\infty}\left(0, T ; L^{2}\left(\mathbb{R}_{+}^{3}\right)\right)},\|\nabla u\|_{L^{2}\left(0, T ; L^{2}\left(\mathbb{R}_{+}^{3}\right)\right)},\left\|v_{0}\right\|_{B_{p q, 0}^{1-\frac{2}{q}\left(\mathbb{R}_{+}^{3}\right)}}\right),
\end{aligned}
$$

where $3 / l+2 / m=4$ and $1<m<2$. In the case that $\Omega=\mathbb{R}_{+}^{3}$, however, with the aid of estimates of the Stokes system in Theorem 1.1. we can improve the estimate (1.23) in a half-space. More precisely, we obtain the following:

Theorem 1.3. Let $1<p \leq 3 / 2,1 / p<\beta \leq 1$ and $1<q<2$ with $3 / p+2 / q=3+\beta$. Assume that $v_{0} \in B_{p q, 0}^{1+\beta-\frac{2}{q}}\left(\mathbb{R}_{+}^{3}\right)$ with $\operatorname{div} v_{0}=0$ in the sense of distributions. Suppose that $v$ is a weak solution of the (1.19) and $p$ the associated pressure. Then,

$$
\|v\|_{L^{q}\left(0, T ; H_{p}^{1+\beta}\left(\mathbb{R}_{+}^{3}\right)\right)}+\|p\|_{L^{q}\left(0, T ; H_{p}^{\beta}\left(\mathbb{R}_{+}^{3}\right)\right)} \leq c\left\||v|^{2}\right\|_{L^{q}\left(0, T ; H_{p, 0}^{\beta}\left(\mathbb{R}_{+}^{3}\right)\right)}+c\left\|v_{0}\right\|_{B_{p q, 0}^{1+\beta-\frac{2}{q}}\left(\mathbb{R}_{+}^{3}\right)} .
$$

Furthermore, due to Sobolev imbedding,

$$
\|p\|_{L^{q}\left(0, T ; \tilde{p}^{\tilde{p}}\left(\mathbb{R}_{+}^{3}\right)\right)} \leq c\left\||v|^{2}\right\|_{L^{q}\left(0, T ; H_{p, 0}^{\beta}\left(\mathbb{R}_{+}^{3}\right)\right)}+c\left\|v_{0}\right\|_{B_{p q, 0}^{1+\beta-\frac{2}{q}}\left(\mathbb{R}_{+}^{3}\right)},
$$

where $1 / \tilde{p}=1 / p-\beta / 3$ and $3 / \tilde{p}+2 / q=3$. The righthand sides in (1.24) and (1.25) are bounded by $C=C\left(\|u\|_{L^{\infty}\left(0, T ; L^{2}\left(\mathbb{R}_{+}^{3}\right)\right)},\|\nabla u\|_{L^{2}\left(0, T ; L^{2}\left(\mathbb{R}_{+}^{3}\right)\right)},\left\|v_{0}\right\|_{B_{p q, 0}^{1-\frac{2}{q}}\left(\mathbb{R}_{+}^{3}\right)}\right)$.

The proof of Theorem 1.3 will be given in section 5. This paper is organized as follows. In section 2, we introduce some function spaces. Theorem 1.1 will be proved in section 3 and section 4 by treating non-homogeneous case and non-zero initial data separately. Section 5 is devoted to proofs of Theorem 1.2 and Theorem 1.3

\section{Preliminaries}

In this section, we recall some function spaces, remind some known results used later and provide proofs of preliminary results useful for our purpose. We start with introducing function spaces. As a notational convenience, if there exists a constant $c>0$ such that $c A \leq B \leq c^{-1} A$, then we write $A \approx B$. 


\subsection{Function spaces}

- (Sobolev and Besov spaces in $\mathbb{R}^{n}$ ) For $\alpha \in \mathbb{R}$, we consider distributions $g_{\alpha}$ and $G_{\alpha}$, whose Fourier transforms in $\mathbb{R}^{n}$ are given as follows: For $\xi \in \mathbb{R}^{n}$

$$
\widehat{G_{\alpha}}(\xi)=\left(1+4 \pi^{2}|\xi|^{2}\right)^{-\frac{\alpha}{2}}, \quad \widehat{g_{\alpha}}(\xi)=\left(4 \pi^{2}|\xi|^{2}\right)^{-\frac{\alpha}{2}} .
$$

For $\alpha \in \mathbb{R}$ and $1 \leq p \leq \infty$ we define the generalized Sobolev space $H_{p}^{\alpha}\left(\mathbb{R}^{n}\right)$ and the homogeneous Sobolev space $\dot{H}_{p}^{\alpha}\left(\mathbb{R}^{n}\right)$ by

$$
\begin{aligned}
& H_{p}^{\alpha}\left(\mathbb{R}^{n}\right)=\left\{f \in \mathcal{S}^{\prime}\left(\mathbb{R}^{n}\right) \mid\|f\|_{H_{p}^{\alpha}\left(\mathbb{R}^{n}\right)}:=\left\|G_{-\alpha} * f\right\|_{L^{p}\left(\mathbb{R}^{n}\right)}<\infty\right\}, \\
& \dot{H}_{p}^{\alpha}\left(\mathbb{R}^{n}\right)=\left\{f \in \mathcal{S}^{\prime}\left(\mathbb{R}^{n}\right) \mid\|f\|_{\dot{H}_{p}^{\alpha}\left(\mathbb{R}^{n}\right)}:=\left\|g_{-\alpha} * f\right\|_{L^{p}\left(\mathbb{R}^{n}\right)}<\infty\right\},
\end{aligned}
$$

where $*$ is a convolution in $\mathbb{R}^{n}$ and $\mathcal{S}^{\prime}\left(\mathbb{R}^{n}\right)$ is the dual space of the Schwartz space $\mathcal{S}\left(\mathbb{R}^{n}\right)$. We note that for non-negative integer $k$ and $1 \leq p \leq \infty$,

$$
\begin{aligned}
& H_{p}^{k}\left(\mathbb{R}^{n}\right)=\left\{f \in L^{p}\left(\mathbb{R}^{n}\right) \mid \sum_{0 \leq l \leq k} D_{x}^{l} f \in L^{p}\left(\mathbb{R}^{n}\right)\right\}, \\
& \dot{H}_{p}^{k}\left(\mathbb{R}^{n}\right)=\left\{f \in L_{\mathrm{loc}}^{p}\left(\mathbb{R}^{n}\right) \mid \sum_{l=k} D_{x}^{l} f \in L^{p}\left(\mathbb{R}^{n}\right)\right\} .
\end{aligned}
$$

Let $\alpha \in \mathbb{R}$ and $1 \leq p, q<\infty$. The Besov space and the homogeneous Besov space in $\mathbb{R}^{n}$, denoted by $B_{p q}^{\alpha}\left(\mathbb{R}^{n}\right)$ and $\dot{B}_{p q}^{\alpha}\left(\mathbb{R}^{n}\right)$, are defined as follows, respectively:

$$
\begin{aligned}
& B_{p q}^{\alpha}\left(\mathbb{R}^{n}\right)=\left\{f \mid\|f\|_{B_{p q}^{\alpha}\left(\mathbb{R}^{n}\right)}:=\|f * \psi\|_{L^{p}}+\left[\sum_{1 \leq k<\infty}\left(2^{\alpha k}\left\|f * \phi_{k}\right\|_{L^{p}}\right)^{q}\right]^{\frac{1}{q}}<\infty\right\}, \\
& \dot{B}_{p q}^{\alpha}\left(\mathbb{R}^{n}\right)=\left\{f \mid\|f\|_{\dot{B}_{p q}^{\alpha}\left(\mathbb{R}^{n}\right)}:=\left[\sum_{-\infty<k<\infty}\left(2^{\alpha k}\left\|f * \phi_{k}\right\|_{\left.L^{p}\right)^{q}}\right]^{\frac{1}{q}}<\infty\right\},\right.
\end{aligned}
$$

where $\psi$ and $\phi$ are functions in Schwartz space in $\mathbb{R}^{n}$ such that $\phi_{k}(x)=2^{k n} \phi\left(2^{k} x\right)$ with $\operatorname{supp} \hat{\phi}(\xi)=$ $\left\{\xi\left|2^{-2}<\right| \xi \mid<2^{2}\right\}$ and

$$
\hat{\psi}(\xi)+\sum_{1 \leq k<\infty} \hat{\phi}\left(2^{-k} \xi\right)=1 \text { for } \xi \in \mathbb{R}^{n}, \quad \sum_{-\infty<k<\infty} \hat{\phi}\left(2^{-k} \xi\right)=1 \text { for } \xi \neq 0 .
$$

The following properties of complex interpolation and norm equivalence can be found in 2 , Theorem 6.3.2, Theorem 6.4.5] and [1, Theorem 4.17].

Proposition 2.1. Let $1<p, q<\infty$ and $\alpha_{1}, \alpha, \alpha_{2} \in \mathbb{R}$ with $\alpha_{1} \leq \alpha \leq \alpha_{2}$.

(i) $\dot{H}_{p}^{\alpha}\left(\mathbb{R}^{n}\right) \cap L^{p}\left(\mathbb{R}^{n}\right)=H_{p}^{\alpha}\left(\mathbb{R}^{n}\right)$ and $\|f\|_{H_{p}^{\alpha}} \approx\|f\|_{L^{p}}+\|f\|_{\dot{H}_{p}^{\alpha}}$ for $\alpha>0$.

(ii) (Complex interpolation) Let $\theta \in(0,1)$ with $\alpha=(1-\theta) \alpha_{0}+\theta \alpha_{1}$.

$$
\begin{aligned}
{\left[H_{p}^{\alpha_{0}}, H_{p}^{\alpha_{1}}\right]_{\theta} } & =H_{p}^{\alpha}, & {\left[\dot{H}_{p}^{\alpha_{0}}, \dot{H}_{p}^{\alpha_{1}}\right]_{\theta} } & =\dot{H}_{p}^{\alpha}, \\
{\left[B_{p q}^{\alpha_{0}}, B_{p q}^{\alpha_{1}}\right]_{\theta} } & =B_{p q}^{\alpha}, & {\left[\dot{B}_{p q}^{\alpha_{0}}, \dot{B}_{p q}^{\alpha_{1}}\right]_{\theta} } & =\dot{B}_{p q}^{\alpha}
\end{aligned}
$$

(iii) Let $k<\alpha<k+1$ for non-negative integer $k$. Then,

$$
\|f\|_{B_{p q}^{\alpha}\left(\mathbb{R}^{n}\right)} \approx\|f\|_{H_{p}^{k}\left(\mathbb{R}^{n}\right)}+\sum_{|\beta|=k}\left(\int_{\mathbb{R}^{n}}\left(\int_{\mathbb{R}^{n}} \frac{\left|D^{\beta} f(x)-D^{\beta} f(y)\right|^{p}}{|x-y|^{n+\frac{p}{q} \alpha}} d y\right)^{\frac{q}{p}} d x\right)^{\frac{1}{q}},
$$




$$
\|f\|_{\dot{B}_{p q}^{\alpha}\left(\mathbb{R}^{n}\right)} \approx \sum_{|\beta|=k}\left(\int_{\mathbb{R}^{n}}\left(\int_{\mathbb{R}^{n}} \frac{\left|D^{\beta} f(x)-D^{\beta} f(y)\right|^{p}}{|x-y|^{n+\frac{p}{q} \alpha}} d y\right)^{\frac{q}{p}} d x\right)^{\frac{1}{q}},
$$

where $\beta=\left(\beta_{1}, \beta_{2}, \cdots, \beta_{n}\right) \in(\mathbb{N} \cup\{0\})^{n}$ and $D^{\beta}=D_{x_{1}}^{\beta_{1}} D_{x_{2}}^{\beta_{2}} \cdots D_{x_{n}}^{\beta_{n}}$.

- (Sobolev space, Besov space and their dual spaces in $\mathbb{R}_{+}^{n}$ ) Let $r f$ be a restriction over $\mathbb{R}_{+}^{n}$ of the function $f$ defined in $\mathbb{R}^{n}$. For $\alpha \geq 0$, we define function spaces in a half space as follows:

$$
\begin{array}{ll}
H_{p}^{\alpha}\left(\mathbb{R}_{+}^{n}\right):=\left\{r f \mid f \in H_{p}^{\alpha}\left(\mathbb{R}^{n}\right)\right\}, & \dot{H}_{p}^{\alpha}\left(\mathbb{R}_{+}^{n}\right):=\left\{r f \mid f \in \dot{H}_{p}^{\alpha}\left(\mathbb{R}^{n}\right)\right\}, \\
B_{p q}^{\alpha}\left(\mathbb{R}_{+}^{n}\right):=\left\{r f \mid f \in B_{p q}^{\alpha}\left(\mathbb{R}^{n}\right)\right\}, & \dot{B}_{p q}^{\alpha}\left(\mathbb{R}_{+}^{n}\right):=\left\{r f \mid f \in \dot{B}_{p q}^{\alpha}\left(\mathbb{R}^{n}\right)\right\}
\end{array}
$$

with their norms $\|f\|_{X\left(\mathbb{R}_{+}^{n}\right)}:=\inf \|F\|_{X\left(\mathbb{R}^{n}\right)}$ for all $F \in X\left(\mathbb{R}^{n}\right)$ with $r F=f$, where $X=H_{p}^{\alpha}, \dot{H}_{p}^{\alpha}, B_{p q}^{\alpha}$ and $\dot{B}_{p q}^{\alpha}$. Here we note that for non-negative integer $k$ and $1<p<\infty$ (see [14, Chapter 2])

$$
\begin{aligned}
& H_{p}^{k}\left(\mathbb{R}_{+}^{n}\right)=\left\{f \mid \sum_{0 \leq l \leq k}\left\|D_{x}^{l} f\right\|_{L^{p}\left(\mathbb{R}_{+}^{n}\right)}<\infty\right\}, \\
& \dot{H}_{p}^{k}\left(\mathbb{R}_{+}^{n}\right)=\left\{f \mid \sum_{l=k}\left\|D_{x}^{l} f\right\|_{L^{p}\left(\mathbb{R}_{+}^{n}\right)}<\infty\right\} .
\end{aligned}
$$

Following arguments in [14, Chapter 2], for $\alpha>0$ and integers $k_{1}, k_{2} \geq 0$ we observe that

$$
\left[H_{p}^{k_{1}}\left(\mathbb{R}_{+}^{n}\right), H_{p}^{k_{2}}\left(\mathbb{R}_{+}^{n}\right)\right]_{\theta}=H_{p}^{\alpha}\left(\mathbb{R}_{+}^{n}\right), \quad\left[\dot{H}_{p}^{k_{1}}\left(\mathbb{R}_{+}^{n}\right), \dot{H}_{p}^{k_{2}}\left(\mathbb{R}_{+}^{n}\right)\right]_{\theta}=\dot{H}_{p}^{\alpha}\left(\mathbb{R}_{+}^{n}\right),
$$

where $\alpha=\theta k_{1}+(1-\theta) k_{2}$. For later use, we recall an interpolation result in [31, section 1.18.4].

Proposition 2.2. Let $I=(0, T), \alpha_{1}, \alpha_{2} \in \mathbb{R}, 0<\theta<1, \alpha=\theta \alpha_{1}+(1-\theta) \alpha_{2}$ and $1<p<\infty$.

$$
\left[L^{p}\left(I ; X_{1}\right), L^{p}\left(I ; X_{2}\right)\right]_{\theta}=L^{p}\left(I ;\left[X_{1} ; X_{2}\right]_{\theta}\right),
$$

where $\left(X_{1}, X_{2}\right):=\left(H_{p}^{\alpha_{1}}\left(\mathbb{R}_{+}^{n}\right), H_{p}^{\alpha_{2}}\left(\mathbb{R}_{+}^{n}\right)\right)$ or $\left(X_{1}, X_{2}\right):=\left(\dot{H}_{p}^{\alpha_{1}}\left(\mathbb{R}_{+}^{n}\right), \dot{H}_{p}^{\alpha_{2}}\left(\mathbb{R}_{+}^{n}\right)\right)$.

We denote by $H_{p, 0}^{-\alpha}\left(\mathbb{R}_{+}^{n}\right), \dot{H}_{p, 0}^{-\alpha}\left(\mathbb{R}_{+}^{n}\right), B_{p q, 0}^{-\alpha}\left(\mathbb{R}_{+}^{n}\right)$ and $\dot{B}_{p q, 0}^{-\alpha}\left(\mathbb{R}_{+}^{n}\right)$ the dual spaces of $H_{p^{\prime}}^{\alpha}\left(\mathbb{R}_{+}^{n}\right)$, $\dot{H}_{p^{\prime}}^{\alpha}\left(\mathbb{R}_{+}^{n}\right), B_{p^{\prime} q^{\prime}}^{\alpha}\left(\mathbb{R}_{+}^{n}\right)$ and $\dot{B}_{p^{\prime} q^{\prime}}^{\alpha}\left(\mathbb{R}_{+}^{n}\right)$ with $\frac{1}{p}+\frac{1}{p^{\prime}}=1$ and $\frac{1}{q}+\frac{1}{q^{\prime}}=1$, namely

$$
\begin{gathered}
H_{p, 0}^{-\alpha}\left(\mathbb{R}_{+}^{n}\right)=\left(H_{p^{\prime}}^{\alpha}\left(\mathbb{R}_{+}^{n}\right)\right)^{\prime}, \quad \dot{H}_{p, 0}^{-\alpha}\left(\mathbb{R}_{+}^{n}\right)=\left(\dot{H}_{p^{\prime}}^{\alpha}\left(\mathbb{R}_{+}^{n}\right)\right)^{\prime}, \\
B_{p q, 0}^{-\alpha}\left(\mathbb{R}_{+}^{n}\right)=\left(B_{p^{\prime} q^{\prime}}^{\alpha}\left(\mathbb{R}_{+}^{n}\right)\right)^{\prime}, \quad \dot{B}_{p q, 0}^{-\alpha}\left(\mathbb{R}_{+}^{n}\right)=\left(\dot{B}_{p^{\prime} q^{\prime}}^{\alpha}\left(\mathbb{R}_{+}^{n}\right)\right)^{\prime} .
\end{gathered}
$$

Here we recall some results of trace theorem between Sobolev spaces and Besov spaces.

Proposition 2.3. (i) If $w \in H_{p}^{\alpha}\left(\mathbb{R}_{+}^{n}\right)$ with $\alpha>\frac{1}{p}$, then $\left.w\right|_{x_{n}=0} \in B_{p}^{\alpha-\frac{1}{p}}\left(\mathbb{R}^{n-1}\right)$ and

$$
\left\|\left.w\right|_{x_{n}=0}\right\|_{B_{p}^{\alpha-\frac{1}{p}}\left(\mathbb{R}^{n-1}\right)} \leq c\|w\|_{H_{p}^{\alpha}\left(\mathbb{R}_{+}^{n}\right)} .
$$

(ii) If $w \in \dot{H}_{p}^{\alpha}\left(\mathbb{R}_{+}^{n}\right)$ with $\alpha>\frac{1}{p}$, then $\left.w\right|_{x_{n}=0} \in \dot{B}_{p}^{\alpha-\frac{1}{p}}\left(\mathbb{R}^{n-1}\right)$ and

$$
\left\|\left.w\right|_{x_{n}=0}\right\|_{\dot{B}_{p}^{\alpha-\frac{1}{p}}\left(\mathbb{R}^{n-1}\right)} \leq c\|w\|_{\dot{H}_{p}^{\alpha}\left(\mathbb{R}_{+}^{n}\right)} .
$$

If $w \in \dot{H}_{p}^{\alpha}\left(\mathbb{R}_{+}^{n}\right)$ with $\alpha \geq 0$ and $w$ is harmonic in $\mathbb{R}_{+}^{n}$, then $\left.w\right|_{x_{n}=0} \in \dot{B}_{p}^{\alpha-\frac{1}{p}}\left(\mathbb{R}^{n-1}\right)$ and

$$
\left\|\left.w\right|_{x_{n}=0}\right\|_{\dot{B}_{p}^{\alpha-\frac{1}{p}}\left(\mathbb{R}^{n-1}\right)} \approx\|w\|_{\dot{H}_{p}^{\alpha}\left(\mathbb{R}_{+}^{n}\right)} .
$$


We remark that the non-homogeneous case with $\alpha>1 / p$ was treated in [15] and the estimate (2.6) of homogeneous case was shown in [13. When $w$ is harmonic and $\alpha=0$, (2.7) was proved in [17, Lemma 2.1]. Via the argument of interpolations, we obtain (2.7) for general case $\alpha>0$.

- (Mixed anisotropic Sobolev spaces) Let $0<\sigma \leq 1$. For $f \in C_{0}^{\infty}(\mathbb{R})$, we introduce a functional given as follows:

$$
I_{\sigma} f(t)=\frac{1}{\Gamma(\sigma)} \int_{-\infty}^{t} \frac{f(s)}{(t-s)^{1-\sigma}} d s, \quad-\infty<t<\infty,
$$

where $\Gamma(\sigma)$ is a Gamma function. The $\sigma$-th fractional derivative of $f, D_{t}^{\sigma} f$, can be defined by

$$
D_{t}^{\sigma} f(t)=\frac{d}{d t} I_{1-\sigma} f(t) \quad \text { for } \quad 0<\sigma<1 \quad \text { and } \quad D_{t}^{1} f(t)=\frac{d}{d t} f(t) .
$$

By the simple computation, we have

$$
I_{\sigma_{1}}\left(I_{\sigma_{2}} f\right)=I_{\sigma_{1}+\sigma_{2}} f, \quad D_{t}^{\sigma_{1}}\left(D_{t}^{\sigma_{2}} f\right)=D_{t}^{\sigma_{1}+\sigma_{2}} f
$$

for $0<\sigma_{1}, \sigma_{2}<1, \sigma_{1}+\sigma_{2} \leq 1$ and $f \in C_{0}^{\infty}(\mathbb{R})$. Furthermore, we have

$$
\widehat{I_{\sigma} f}(\tau)=m_{\sigma}(\tau) \hat{f}(\tau), \quad m_{\sigma}(\tau)=\left(a_{\sigma}-i b_{\sigma} \operatorname{sign}(\tau)\right)|\tau|^{-\sigma},
$$

where

$$
a_{\sigma}=\int_{0}^{\infty} \frac{\cos t}{t^{\sigma}} d t, \quad b_{\sigma}=\int_{0}^{\infty} \frac{\sin t}{t^{\sigma}} d t
$$

Therefore, we note that

$$
\widehat{D_{t}^{\sigma} f}(\tau)=i \tau m_{1-\sigma}(\tau) \hat{f}(\tau)=i\left(a_{1-\sigma}-i b_{1-\sigma} \operatorname{sign}(\tau)\right) \operatorname{sign}(\tau)|\tau|^{\sigma} \hat{f}(\tau) .
$$

We also define the dual operator $J_{\sigma}$ of $I_{\sigma}$ as follows:

$$
J_{\sigma} f(t)=\frac{1}{\Gamma(\sigma)} \int_{t}^{\infty} \frac{f(s)}{(s-t)^{1-\sigma}} d s, \quad D_{t}^{* \sigma} f(t)=D_{t} J_{1-\sigma} f(t)
$$

such that

$$
\int_{\mathbb{R}} I_{\sigma} f(t) g(t) d t=\int_{\mathbb{R}} f(t) J_{\sigma} g(t) d t, \quad \int_{\mathbb{R}} D_{t}^{\sigma} f(t) g(t) d t=-\int_{\mathbb{R}} f(t) D_{t}^{* \sigma} g(t) d t .
$$

Using the Plancherel theorem, we get $\widehat{J_{\sigma}} g(\tau)=\bar{m}_{\sigma}(\tau) \hat{g}(\tau)$, where $\bar{m}_{\sigma}$ is the complex conjugate of $m_{\sigma}$ in (2.9). Rewriting $\bar{m}_{\sigma}(\tau)=m_{\sigma}(\tau) \frac{\bar{m}_{\sigma}(\tau)}{m_{\sigma}(\tau)}$ and noting that

$$
\frac{\bar{m}_{\sigma}(\tau)}{m_{\sigma}(\tau)}=\frac{a_{\sigma}^{2}-b_{\sigma}^{2}+2 i a_{\sigma} b_{\sigma} \operatorname{sign}(\tau)}{a_{\sigma}^{2}+b_{\sigma}^{2}}
$$

we get

$$
J_{\sigma} g(t)=I_{\sigma}\left(A_{\sigma} g+B_{\sigma} H g\right)(t), \quad D_{t}^{\sigma *} g(t)=D_{t}^{\sigma}\left(A_{1-\sigma} g+B_{1-\sigma} H g\right)(t),
$$

where $A_{\sigma}=\left(a_{\sigma}^{2}-b_{\sigma}^{2}\right) /\left(a_{\sigma}^{2}+b_{\sigma}^{2}\right), B_{\sigma}=\left(2 a_{\sigma} b_{\sigma}\right) /\left(a_{\sigma}^{2}+b_{\sigma}^{2}\right)$ and $H$ is the Hilbert transform. Next, we define the mixed anisotropic Sobolev spaces $H_{p q}^{\alpha, \frac{1}{2} \alpha}\left(\mathbb{R}^{n+1}\right)$ and $\dot{H}_{p q}^{\alpha, \frac{1}{2} \alpha}\left(\mathbb{R}^{n+1}\right)$ with $0<\alpha \leq 2$ as follows:

$$
\begin{aligned}
& H_{p q}^{\alpha, \frac{1}{2} \alpha}\left(\mathbb{R}^{n+1}\right):=\left\{f \in L_{t}^{q} L_{x}^{p} \mid\|f\|_{H_{p q}^{\alpha, \frac{1}{2} \alpha}\left(\mathbb{R}^{n+1}\right)}^{q}:=\int_{\mathbb{R}}\left(\left\|D_{t}^{\frac{1}{2} \alpha} f\right\|_{L^{p}\left(\mathbb{R}^{n}\right)}^{q}+\|f\|_{H_{p}^{\alpha}\left(\mathbb{R}^{n}\right)}^{q}\right) d t<\infty\right\}, \\
& \dot{H}_{p q}^{\alpha, \frac{1}{2} \alpha}\left(\mathbb{R}^{n+1}\right):=\left\{f \in L_{l o c}^{1}\left(\mathbb{R}^{n+1}\right) \mid\|f\|_{\dot{H}_{p q}^{\alpha, \frac{1}{2} \alpha}\left(\mathbb{R}^{n+1}\right)}^{q}:=\int_{\mathbb{R}}\left(\left\|D_{t}^{\frac{1}{2} \alpha} f\right\|_{L^{p}\left(\mathbb{R}^{n}\right)}^{q}+\|f\|_{\dot{H}_{p}^{\alpha}\left(\mathbb{R}^{n}\right)}^{q}\right) d t<\infty\right\} .
\end{aligned}
$$


Note that if $\operatorname{supp} f \subset[0, T)$ (so that $f(T)=0$ ), then

$$
J_{\sigma} f(t)=\frac{1}{\Gamma(\sigma)} \int_{t}^{T} \frac{f(s)}{(s-t)^{1-\sigma}} d s
$$

Let $I=(0, T)$ and we denote $Q_{T}=\mathbb{R}^{n} \times I, Q_{T}^{+}=\mathbb{R}_{+}^{n} \times I$, unless any confusion is to be expected. Here we define the mixed anisotropic Sobolev spaces $H_{p q, *}^{\alpha, \frac{1}{2} \alpha}\left(Q_{T}^{+}\right)$and $\dot{H}_{p q, *}^{\alpha, \frac{1}{2} \alpha}\left(Q_{T}^{+}\right), 0<\alpha \leq 2$ in the following way: We say that $f$ is in $H_{p q, *}^{\alpha, \frac{1}{2} \alpha}\left(Q_{T}^{+}\right)\left(f\right.$ is in $\left.\dot{H}_{p q, *}^{\alpha, \frac{1}{2} \alpha}\left(Q_{T}^{+}\right)\right)$means $\operatorname{supp} f \subset \overline{\mathbb{R}_{+}^{n}} \times[0, T)$ and

$$
\begin{gathered}
\|f\|_{H_{p, *}^{\alpha, \frac{1}{2} \alpha}\left(Q_{T}^{+}\right)}^{q}:=\int_{I}\left(\left\|D_{t}^{* \frac{1}{2} \alpha} f\right\|_{L^{p}\left(\mathbb{R}_{+}^{n}\right)}^{q}+\|f\|_{H_{p}^{\alpha}\left(\mathbb{R}_{+}^{n}\right)}^{q}\right) d t<\infty \\
\left(\|f\|_{\dot{H}_{p,+*}^{\alpha, \frac{1}{2} \alpha}\left(Q_{T}^{+}\right)}^{q}:=\int_{I}\left(\left\|D_{t}^{* \frac{1}{2} \alpha} f\right\|_{L^{p}\left(\mathbb{R}_{+}^{n}\right)}^{q}+\|f\|_{\dot{H}_{p}^{\alpha}\left(\mathbb{R}_{+}^{n}\right)}^{q}\right) d t<\infty\right) .
\end{gathered}
$$

We mean by $H_{p^{\prime} q^{\prime}, 0}^{-\alpha,-\frac{1}{2} \alpha}\left(Q_{T}^{+}\right)$and $\dot{H}_{p^{\prime} q^{\prime}, 0}^{-\alpha, \frac{1}{2} \alpha}\left(Q_{T}^{+}\right)$the dual spaces of $H_{p q, *}^{\alpha, \frac{1}{2} \alpha}\left(Q_{T}^{+}\right)$and $\dot{H}_{p q, *}^{\alpha, \frac{1}{2} \alpha}\left(Q_{T}^{+}\right)$, where $\frac{1}{p}+\frac{1}{p^{\prime}}=1$ and $\frac{1}{q}+\frac{1}{q^{\prime}}=1$. Similarly, we define function spaces $H_{p q, *}^{\alpha, \frac{1}{2} \alpha}\left(Q_{T}\right), \dot{H}_{p q, *}^{\alpha, \frac{1}{2} \alpha}\left(Q_{T}\right)$ and their dual spaces $H_{p^{\prime} q^{\prime}, 0}^{-\alpha, \frac{1}{2} \alpha}\left(Q_{T}\right), \dot{H}_{p^{\prime} q^{\prime}, 0}^{-\alpha, \frac{1}{2} \alpha}\left(Q_{T}\right)$, respectively.

Remark We note that $L^{q}\left(I ; C_{c}^{\infty}\left(\mathbb{R}^{n}\right)\right)$ is dense subset of $H_{p q, 0}^{-\alpha,-\frac{1}{2} \alpha}\left(Q_{T}\right)$ and $\dot{H}_{p q, 0}^{-\alpha,-\frac{1}{2} \alpha}\left(Q_{T}\right)$. We also remark that $\dot{H}_{p q, *}^{\alpha, \frac{1}{2} \alpha}\left(\mathbb{R}_{+}^{n} \times I\right) \subset L^{q}\left(I ; \dot{H}_{p}^{\alpha}\left(\mathbb{R}_{+}^{n}\right)\right), L^{q}\left(I ; \dot{H}_{p}^{-\alpha}\left(\mathbb{R}_{+}^{n}\right)\right) \subset \dot{H}_{p q, 0}^{-\alpha,-\frac{1}{2} \alpha}\left(\mathbb{R}_{+}^{n} \times I\right)$ and

$$
\|f\|_{\dot{H}_{p q, 0}^{-\alpha,-\frac{1}{2} \alpha}\left(\mathbb{R}_{+}^{n} \times I\right)} \leq\|f\|_{L_{t}^{q}\left(I ; \dot{H}_{p}^{-\alpha}\left(\mathbb{R}_{+}^{n}\right)\right)} .
$$

For $\phi \in \dot{H}_{p q *}^{\alpha, \frac{1}{2} \alpha}\left(Q_{T}\right)\left(\phi \in H_{p q *}^{\alpha, \frac{1}{2} \alpha}\left(Q_{T}\right)\right)$, we define $\tilde{\phi} \in \dot{H}_{p q}^{\alpha, \frac{1}{2} \alpha}\left(\mathbb{R}^{n+1}\right)\left(\tilde{\phi} \in H_{p q}^{\alpha, \frac{1}{2} \alpha}\left(\mathbb{R}^{n+1}\right)\right)$ by

$$
\tilde{\phi}(x, t)= \begin{cases}\phi(x, t), & x \in \mathbb{R}^{n}, 0<t<T, \\ \phi(x,-t), & x \in \mathbb{R}^{n},-T<t<0, \\ 0, & \text { otherwise }\end{cases}
$$

and it is direct that $\|\tilde{\phi}\|_{\dot{H}_{p q}^{\alpha, \frac{1}{2} \alpha}\left(\mathbb{R}^{n+1}\right)} \leq c\|\phi\|_{\dot{H}_{p q *}^{\alpha, \frac{1}{2} \alpha}\left(Q_{T}\right)}\left(\|\tilde{\phi}\|_{H_{p q}^{\alpha, \frac{1}{2} \alpha}\left(\mathbb{R}^{n+1}\right)} \leq c\|\phi\|_{H_{p q *}^{\alpha, \frac{1}{2} \alpha}\left(Q_{T}\right)}\right)$. Next proposition shows that fractional derivatives are bounded operators between anisotropic Sobolev spaces.

Proposition 2.4. Let $0 \leq \sigma_{1}, \sigma_{2} \leq 1$ and $\sigma_{1}+\sigma_{2} \leq 1$.

(i) $D_{t}^{\frac{1}{2} \sigma_{1}}$ and $\Delta^{\frac{1}{2} \sigma_{1}}$ are bounded operators from $\dot{H}_{p q}^{\sigma_{1}+\sigma_{2}, \frac{1}{2} \sigma_{1}+\frac{1}{2} \sigma_{2}}\left(\mathbb{R}^{n+1}\right)$ to $\dot{H}_{p q}^{\sigma_{2}, \frac{1}{2} \sigma_{2}}\left(\mathbb{R}^{n+1}\right)$.

(ii) $D_{t}^{* \frac{1}{2} \sigma_{1}}$ and $\Delta^{\frac{1}{2} \sigma_{1}}$ are bounded operators from $\dot{H}_{p q *}^{\sigma_{1}+\sigma_{2}, \frac{1}{2} \sigma_{1}+\frac{1}{2} \sigma_{2}}\left(Q_{T}\right)$ to $\dot{H}_{p q *}^{\sigma_{2}, \frac{1}{2} \sigma_{2}}\left(Q_{T}\right)$.

Proof. We recall by definition that

$$
\left\|D_{t}^{\frac{1}{2} \sigma_{1}} f\right\|_{\dot{H}_{p q}^{\sigma_{2}, \frac{1}{2} \sigma_{2}}}^{q}=\int_{\mathbb{R}}\left(\left\|D_{t}^{\frac{1}{2} \sigma_{2}} D_{t}^{\frac{1}{2} \sigma_{1}} f\right\|_{L^{p}\left(\mathbb{R}^{n}\right)}^{q}+\left\|\Delta^{\frac{1}{2} \sigma_{2}} D_{t}^{\frac{1}{2} \sigma_{1}} f\right\|_{L^{p}\left(\mathbb{R}^{n}\right)}^{q}\right) d t
$$

Since $D_{t}^{\frac{1}{2} \sigma_{2}} D_{t}^{\frac{1}{2} \sigma_{1}} f=D_{t}^{\frac{1}{2} \sigma_{1}+\frac{1}{2} \sigma_{2}} f$, the first term in (2.11) is dominated by $\|f\|_{\dot{H}_{p q}^{\sigma_{1}+\sigma_{2}, \frac{1}{2} \sigma_{1}+\frac{1}{2} \sigma_{2}}}^{q}$. On the other hand, we note that

$$
\mathcal{F}\left(\Delta^{\frac{1}{2} \sigma_{2}} D_{t}^{\sigma_{1}} f\right)(\xi, \tau)=i \tau|\xi|^{\sigma_{2}} m_{1-\sigma_{1}}(\tau) \hat{f}(\xi, \tau)
$$




$$
=\frac{i \tau|\xi|^{\sigma_{2}} m_{1-\sigma_{1}}(\tau)}{|\xi|^{\sigma_{1}+\sigma_{2}}+i \tau m_{1-\sigma_{1}-\sigma_{2}}(\tau)}\left(|\xi|^{\sigma_{1}+\sigma_{2}}+i \tau m_{1-\sigma_{1}-\sigma_{2}}(\tau)\right) \hat{f}
$$

Here, $\mathcal{F}$ is the Fourier transform in $\mathbb{R}^{n+1}$. We note that $\frac{i \tau|\xi|^{\sigma_{2}} m_{1-\sigma_{1}}(\tau)}{|\xi|^{\sigma_{1}+\sigma_{2}}+i \tau m_{1-\sigma_{1}-\sigma_{2}}(\tau)}$ is a $L_{t}^{q} L_{x}^{p}$-multiplier (see e.g. [20, Theorem A]), and thus the term in (2.12) is dominated by $\|f\|_{\dot{H}_{p q}^{\sigma_{1}}+\sigma_{2}, \frac{1}{2} \sigma_{1}+\frac{1}{2} \sigma_{2}}^{q}$. Hence, $D^{\frac{1}{2} \sigma_{1}}$ is a bounded operators from $\dot{H}_{p q}^{\sigma_{1}+\sigma_{2}, \frac{1}{2} \sigma_{1}+\frac{1}{2} \sigma_{2}}$ to $\dot{H}_{p q}^{\sigma_{2}, \frac{1}{2} \sigma_{2}}$. Similarly, we can see that $\Delta^{\frac{1}{2} \sigma_{1}}$ bounded operators from $\dot{H}_{p q}^{\sigma_{1}+\sigma_{2}, \frac{1}{2} \sigma_{1}+\frac{1}{2} \sigma_{2}}$ to $\dot{H}_{p q}^{\sigma_{2}, \frac{1}{2} \sigma_{2}}$. This completes the proof of $(i)$.

For $\phi \in \dot{H}_{p q *}^{\sigma_{1}+\sigma_{2}, \frac{1}{2} \sigma_{1}+\frac{1}{2} \sigma_{2}}\left(Q_{T}\right)$, we let $\tilde{\phi} \in \dot{H}_{p q}^{\sigma_{1}+\sigma_{2}, \frac{1}{2} \sigma_{1}+\frac{1}{2} \sigma_{2}}\left(\mathbb{R}^{n+1}\right)$ as defined in (2.10). Then, via the result (i), we obtain

$$
\left\|D_{t}^{* \frac{1}{2} \sigma_{1}} \tilde{\phi}\right\|_{\dot{H}_{p q}^{\sigma_{2}, \frac{1}{2} \sigma_{2}}\left(\mathbb{R}^{n+1}\right)} \leq c\|\tilde{\phi}\|_{\dot{H}_{p q}^{\sigma_{1}+\sigma_{2}, \frac{1}{2} \sigma_{1}+\frac{1}{2} \sigma_{2}}\left(\mathbb{R}^{n+1}\right)} \leq c\|\phi\|_{\dot{H}_{p q *}^{\sigma_{1}+\sigma_{2}, \frac{1}{2} \sigma_{1}+\frac{1}{2} \sigma_{2}}\left(Q_{T}\right)} .
$$

Since $D_{t}^{* \frac{1}{2} \sigma_{1}} \tilde{\phi}(x, t)=D_{t}^{* \frac{1}{2} \sigma_{1}} \phi(x, t)$ for $(x, t) \in Q_{T}$, we have

$$
\left\|D_{t}^{* \frac{1}{2} \sigma_{1}} \phi\right\|_{\dot{H}_{p q *}^{\sigma_{2}, \frac{1}{2} \sigma_{2}}\left(Q_{T}\right)} \leq c\|\phi\|_{\dot{H}_{p q *}^{\sigma_{1}+\sigma_{2}, \frac{1}{2} \sigma_{1}+\frac{1}{2} \sigma_{2}}\left(Q_{T}\right)} .
$$

This complete the proof of $(i i)$ and thus we deduce the proposition.

Remark With the aid of duality argument, we also conclude that $D_{t}^{\frac{1}{2} \sigma_{1}}$ and $\Delta^{\frac{1}{2} \sigma_{1}}$ are bounded operators from $\dot{H}_{p q, 0}^{-\sigma_{2},-\frac{1}{2} \sigma_{2}}\left(Q_{T}\right)$ to $\dot{H}_{p q, 0}^{-\sigma_{1}-\sigma_{2},-\frac{1}{2} \sigma_{1}-\frac{1}{2} \sigma_{2}}\left(Q_{T}\right)$.

Proposition 2.5. Let $0 \leq \alpha \leq 2, f \in \dot{H}_{p q, 0}^{\alpha-2, \frac{1}{2} \alpha-1}\left(Q_{T}\right)$ and $T f(x, t):=<f, \Gamma(x-\cdot, t-\cdot)>$. Then, Tf $\in \dot{H}_{p q}^{\alpha, \frac{1}{2} \alpha}\left(Q_{T}\right)$ and the following estimate holds:

$$
\|T f\|_{\dot{H}_{p q}^{\alpha, \frac{1}{2} \alpha}\left(Q_{T}\right)} \leq c\|f\|_{\dot{H}_{p q, 0}^{\alpha-2, \frac{1}{2} \alpha-1}\left(Q_{T}\right)} .
$$

Proof. For the case of $f \in L_{x, t}^{p q}\left(Q_{T}\right)$, it was shown in 21] that $T f \in \dot{H}_{p q, 0}^{2,1}\left(Q_{T}\right)$ and

$$
\int_{0}^{T}\left(\left\|D_{x}^{2} T f(\cdot, t)\right\|_{L^{p}\left(\mathbb{R}^{n}\right)}^{q}+\left\|D_{t} T f(\cdot, t)\right\|_{L^{p}\left(\mathbb{R}^{n}\right)}^{q}\right) d t \leq c \int_{0}^{T}\|f(\cdot, t)\|_{L^{p}\left(\mathbb{R}^{n}\right)}^{q} d t .
$$

With the same argument, we define a bounded operator $T^{*}: L^{p q}\left(Q_{T}\right) \rightarrow \dot{H}_{p q^{*}}^{2,1}\left(Q_{T}\right)$ by

$$
T^{*} g(y, s):=\int_{s}^{T} \int_{\mathbb{R}^{n}} \Gamma(x-y, t-s) g(x, t) d x d t .
$$

Since $\dot{H}_{p q, 0}^{-2,-1}\left(Q_{T}\right)=\left(\dot{H}_{p^{\prime} q^{\prime *}}^{2,1}\left(Q_{T}\right)\right)^{*}$, for $f \in L^{q}\left(I ; \mathbb{R}^{n}\right)$, we have via Hölder's equality

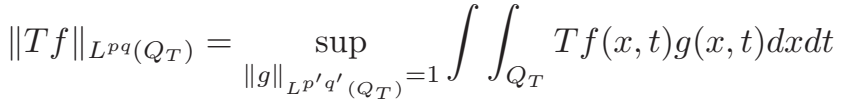

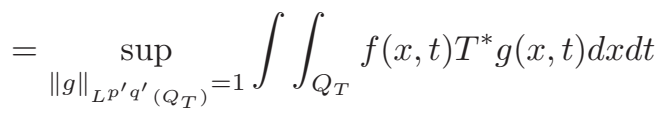

$$
\begin{aligned}
& \leq \sup _{\|g\|_{L^{p^{\prime} q^{\prime}}\left(Q_{T}\right)}=1}\left\|T^{*} g\right\|_{\dot{H}_{p^{\prime} q^{\prime *}}^{2,1}\left(Q_{T}\right)}\|f\|_{H_{p q, 0}^{-2,-1}\left(Q_{T}\right)}
\end{aligned}
$$

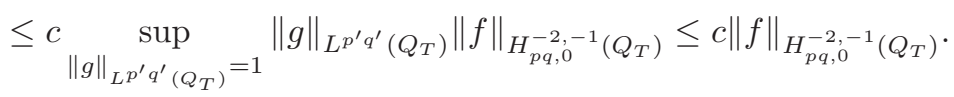


Since $L^{q}\left(I ; \mathbb{R}^{n}\right)$ is dense in $\dot{H}_{p q, 0}^{-2,-1}\left(Q_{T}\right)$, it follows that for all $f \in \dot{H}_{p q, 0}^{-2,-1}\left(Q_{T}\right)$

$$
\|T f\|_{L^{p q}\left(Q_{T}\right)} \leq c\|f\|_{H_{p q, 0}^{-2,-1}\left(Q_{T}\right)} .
$$

Noting that $D_{t}^{\frac{1}{2} \alpha} T f=T D_{t}^{\frac{1}{2} \alpha} f$ and $\Delta^{\frac{1}{2} \alpha} T f=T \Delta^{\frac{1}{2} \alpha} f$, by Proposition 2.4 we get

$$
\begin{aligned}
& \int_{0}^{T}\left\|D_{t}^{\frac{1}{2} \alpha} T f\right\|_{L_{x}^{p}\left(\mathbb{R}^{n}\right)}^{q} d t \leq c\left\|D_{t}^{\frac{1}{2} \alpha} f\right\|_{H_{p q, 0}^{-2,-1}\left(Q_{T}\right)}^{q} \leq c\|f\|_{H_{p q, 0}^{\alpha-2, \frac{1}{2} \alpha-1}\left(Q_{T}\right)}^{q}, \\
& \int_{0}^{T}\left\|\Delta^{\frac{1}{2} \alpha} T f\right\|_{L^{p}\left(\mathbb{R}^{n}\right)}^{q} d t \leq c\left\|\Delta^{\frac{1}{2} \alpha} f\right\|_{H_{p q, 0}^{-2,-1}\left(Q_{T}\right)}^{q} \leq c\|f\|_{H_{p q, 0}^{\alpha-2, \frac{1}{2} \alpha-1}\left(Q_{T}\right)}^{q} .
\end{aligned}
$$

This completes the proof.

Remark For $f \in \dot{H}_{p q, 0}^{-\alpha,-\frac{1}{2} \alpha}\left(Q_{T}^{+}\right)\left(f \in H_{p q, 0}^{-\alpha,-\frac{1}{2} \alpha}\left(Q_{T}^{+}\right)\right)$, we define $\tilde{f} \in \dot{H}_{p q, 0}^{-\alpha,-\frac{1}{2} \alpha}\left(Q_{T}\right)(\tilde{f} \in$ $\left.H_{p q, 0}^{-\alpha,-\frac{1}{2} \alpha}\left(Q_{T}\right)\right)$ by

$$
<\tilde{f}, \phi>=<f,\left.\phi\right|_{Q_{T}^{+}}>, \quad \phi \in \dot{H}_{p q, 0}^{-\alpha,-\frac{1}{2} \alpha}\left(Q_{T}\right)\left(\phi \in H_{p q, 0}^{-\alpha,-\frac{1}{2} \alpha}\left(Q_{T}\right)\right)
$$

and it is immediate that $\|\tilde{f}\|_{\dot{H}_{p q}^{\alpha, \frac{1}{2} \alpha}\left(Q_{T}\right)} \leq c\|f\|_{\dot{H}_{p q *}^{\alpha, \frac{1}{2} \alpha}\left(Q_{T}^{+}\right)}\left(\|\tilde{f}\|_{H_{p q}^{\alpha, \frac{1}{2} \alpha}\left(Q_{T}\right)} \leq c\|f\|_{H_{p q *}^{\alpha, \frac{1}{2} \alpha}\left(Q_{T}^{+}\right)}\right)$. Therefore, by proposition 2.5, we have

$$
\|T \tilde{f}\|_{\dot{H}_{p q}^{\alpha, \frac{1}{2} \alpha}\left(Q_{T}^{+}\right)} \leq\|T \tilde{f}\|_{\dot{H}_{p q}^{\alpha, \frac{1}{2} \alpha}\left(Q_{T}\right)} \leq c\|\tilde{f}\|_{\dot{H}_{p q, 0}^{\alpha-2, \frac{1}{2} \alpha-1}\left(Q_{T}\right)} \leq c\|f\|_{\dot{H}_{p q, 0}^{\alpha-2, \frac{1}{2} \alpha-1}\left(Q_{T}^{+}\right)}
$$

for $f \in \dot{H}_{p q, 0}^{\alpha-2, \frac{1}{2} \alpha-1}\left(Q_{T}^{+}\right)$.

\section{Stokes system with $f \neq 0$ and $v_{0}=0$}

In this section, we consider the Stokes system with nonhomogeneous external force and zero initial data in $\mathbb{R}_{+}^{n} \times(0, \infty)$, namely

$$
\begin{gathered}
v_{t}-\Delta v+\nabla p=f, \quad \operatorname{div} v=0 \quad \text { in } Q_{T}^{+}:=\mathbb{R}_{+}^{n} \times[0, T) \\
v(x, 0)=0 \quad \text { and } \quad v(x, t)=0, \quad x \in \partial \mathbb{R}_{+}^{n}=\mathbb{R}^{n-1} .
\end{gathered}
$$

As mentioned before, we then study the homogeneous Stokes system non-zero initial data, i.e. $f=0$ and $v_{0} \neq 0$ in a half space next section, and by combining both cases we can obtain the desired estimates, since Stokes system is linear. For simplicity, we denote by $E(x)$ and $\Gamma(x, t)$ the fundamental solutions of the Laplace equation and the heat equation, respectively, that is,

$$
E(x)=\frac{1}{2 \pi} \log |x| \quad \text { if } n=2, \quad E(x)=-\frac{1}{(n-2) \omega_{n}|x|^{n-2}} \quad \text { if } n>2,
$$

where $\omega_{n}$ is the area of the unit sphere in $\mathbb{R}^{n}$ and

$$
\Gamma(x, t)=(4 \pi t)^{-\frac{n}{2}} e^{-\frac{|x|^{2}}{4 t}} \chi_{t>0} .
$$

We recall that it was shown in [27] that if the boundary data $f$ is in $L^{q}\left(I ; C_{c}^{\infty}\left(\mathbb{R}_{+}^{n}\right)\right)$, then the system (3.1)-(3.2) has the following solution formulae:

$$
v(x, t)=\int_{0}^{t} \int_{\mathbb{R}_{+}^{n}} \mathcal{G}(x, y, t-\tau) f(y, \tau) d y d \tau
$$




$$
p(x, t)=\int_{0}^{t} \int_{\mathbb{R}_{+}^{n}} \mathcal{P}(x, y, t-\tau) \cdot f(y, \tau) d y d \tau,
$$

where the matrix $\mathcal{G}=\left(G_{i j}\right)_{1 \leq i, j \leq n}$ and the vector $\mathcal{P}=\left(P_{i}\right)_{1 \leq i \leq n}$ are given as

$$
\begin{aligned}
G_{i j}=\delta_{i j}(\Gamma(x-y, t)- & \left.\Gamma\left(x-y^{*}, t\right)\right)+4\left(1-\delta_{j n}\right) \frac{\partial}{\partial x_{j}} \int_{0}^{x_{n}} \int_{\mathbb{R}^{n-1}} \frac{\partial E(x-z)}{\partial x_{i}} \Gamma\left(z-y^{*}, t\right) d z, \\
P_{j}(x, y, t)= & 4\left(1-\delta_{j n}\right) \frac{\partial}{\partial x_{j}}\left[\int_{\mathbb{R}^{n-1}} \frac{\partial E\left(x^{\prime}-z^{\prime}, x_{n}\right)}{\partial x_{n}} \Gamma\left(z^{\prime}-y^{\prime}, y_{n}, t\right) d z^{\prime}\right. \\
& \left.+\int_{\mathbb{R}^{n-1}} E\left(x^{\prime}-z^{\prime}, x_{n}\right) \frac{\partial \Gamma\left(z^{\prime}-y^{\prime}, y_{n}, t\right)}{\partial y_{n}} d z^{\prime}\right] .
\end{aligned}
$$

Here $x^{\prime}$ stands for $\left(x_{1}, \cdots, x_{n-1}\right)$ and $x^{*}=\left(x^{\prime},-x_{n}\right)$ indicates the point symmetric to $x$ with respect to the plane $x_{n}=0$. Since $C_{c}^{\infty}\left(Q_{T}^{+}\right)$is dense subset of $H_{p q, 0}^{\alpha-2,-\frac{1}{2} \alpha-1}\left(Q_{T}^{+}\right)$, we may assume that $f$ is in $C_{c}^{\infty}\left(Q_{T}^{+}\right)$such that the solution $(v, p)$ of system (3.1)-(3.2) are represented by (3.5) and (3.6). Using the formula (3.3) of $v$ and formula (3.4) of $p$, we will prove the following estimates:

$$
\begin{aligned}
& \|v\|_{H_{p q}^{\alpha, \frac{1}{2} \alpha}\left(\mathbb{R}_{+}^{n} \times(0, T)\right)}+\|p\|_{L^{q}\left((0, T) ; H_{p}^{\alpha-1}\left(\mathbb{R}_{+}^{n}\right)\right)} \leq c\|f\|_{H_{p q, 0}^{\alpha-2, \frac{1}{2} \alpha-1}\left(\mathbb{R}_{+}^{n} \times(0, T)\right)}, \\
& \|v\|_{\dot{H}_{p q}^{\alpha, \frac{1}{2} \alpha}\left(\mathbb{R}_{+}^{n} \times(0, T)\right)}+\|p\|_{L^{q}\left((0, T) ; \dot{H}_{p}^{\alpha-1}\left(\mathbb{R}_{+}^{n}\right)\right)} \leq c\|f\|_{\dot{H}_{p q, 0}^{\alpha-2, \frac{1}{2} \alpha-1}\left(\mathbb{R}_{+}^{n} \times(0, T)\right)} .
\end{aligned}
$$

Since the way of proofs for above estimates are similar, we consider only the case of (3.8). We start with the estimate of velocity field.

\subsection{Estimates of velocity fields}

For convenience, for a measurable function $g$ in $\mathbb{R}_{+}^{n}$ we define

$$
\mathcal{I} g\left(x^{\prime}, x_{n}\right):=\int_{\mathbb{R}^{n-1}} E\left(x^{\prime}-y^{\prime}, 0\right) g\left(y^{\prime}, x_{n}\right) d y^{\prime} .
$$

From the formula (3.3) and the functional (3.9), we decompose $v_{i}$ as follows:

$$
v_{i}(x, t)=u_{i}(x, t)+4 \frac{\partial}{\partial x_{i}} w(x, t)-4 \delta_{i n} \mathcal{I} \sum_{1 \leq j \leq n-1} \frac{\partial}{\partial x_{j}} \mathcal{U}^{*} f_{j}(x, t), \quad i=1, \cdots, n,
$$

where $u_{i}, w$ and $\mathcal{U}^{*} f_{j}$ are defined by

$$
\begin{gathered}
u_{i}(x, t):=\int_{0}^{t} \int_{\mathbb{R}_{+}^{n}}\left(\Gamma(x-y, t-\tau)-\Gamma\left(x-y^{*}, t-\tau\right)\right) f_{i}(y, \tau) d y d \tau \\
w(x, t):=\int_{0}^{x_{n}} \int_{\mathbb{R}^{n-1}} E(x-y) \sum_{1 \leq j \leq n-1} \frac{\partial}{\partial y_{j}} \mathcal{U}^{*} f_{j}(y, t) d y \\
\mathcal{U}^{*} f_{j}(x, t):=\int_{0}^{t} \int_{\mathbb{R}_{+}^{n}} \Gamma\left(x-y^{*}, t-\tau\right) f_{j}(y, \tau) d y d \tau .
\end{gathered}
$$

We consider separately the above terms and first estimate $u_{i}$ for $i=1, \cdots, n$.

Lemma 3.1. Let $0 \leq \alpha \leq 2$ and $0<T<\infty$. Suppose that $f \in H_{p q, 0}^{\alpha-2, \frac{\alpha}{2}-1}\left(\mathbb{R}_{+}^{n} \times(0, T)\right)$ with $\operatorname{div} f=0$ in the sense of distributions. If $u_{i}$ is given in (3.11) for $i=1, \cdots, n$, then

$$
\left\|u_{i}\right\|_{\dot{H}_{p q}^{\alpha, \frac{1}{2} \alpha}\left(\mathbb{R}_{+}^{n} \times(0, T)\right)} \leq c\left\|f_{i}\right\|_{\dot{H}_{p q, 0}^{\alpha-2, \frac{1}{2} \alpha-1}\left(\mathbb{R}_{+}^{n} \times(0, T)\right)} .
$$


Proof. Let $I=(0, T)$. We note that since $L^{q}\left(I ; C_{c}^{\infty}\left(\mathbb{R}_{+}^{n}\right)\right)$ is dense subspace of $L^{q}\left(I ; \dot{H}_{p, 0}^{\alpha-2}\left(\mathbb{R}_{+}^{n}\right)\right)$, we assume without loss of generality that $f \in L^{q}\left(I ; C_{c}^{\infty}\left(\mathbb{R}_{+}^{n}\right)\right)$ with $\operatorname{div} f=0$, and we then perform a priori estimates. Let $f \in L^{q}\left(I ; C_{c}^{\infty}\left(\mathbb{R}_{+}^{n}\right)\right)$ and $\tilde{f}$ be a zero extension of $f$. Then, for $(x, t) \in \mathbb{R}_{+}^{n} \times(0, T)$

$$
\begin{aligned}
u_{i}(x, t) & =\int_{0}^{t} \int_{\mathbb{R}^{n}}\left(\Gamma(x-y, t-\tau)-\Gamma\left(x-y^{*}, t-\tau\right)\right) \tilde{f}_{i}(y, \tau) d y d \tau \\
& =\int_{0}^{t} \int_{\mathbb{R}^{n}} \Gamma(x-y, t-\tau)\left(\tilde{f}_{i}\left(y^{\prime}, y_{n}, \tau\right)-\tilde{f}_{i}^{*}\left(y^{\prime}, y_{n}, \tau\right)\right) d y d \tau:=T\left(\tilde{f}-\tilde{f}^{*}\right),
\end{aligned}
$$

where $f^{*}(y)=f\left(y^{\prime},-y_{n}\right)$. Hence, from proposition 2.5, we have

$$
\begin{gathered}
\left\|u_{i}\right\|_{\dot{H}_{p q}^{\alpha, \frac{1}{2} \alpha}\left(\mathbb{R}_{+}^{n} \times(0, T)\right)}=\left\|T\left(\tilde{f}_{i}-\tilde{f}_{i}^{*}\right)\right\|_{\dot{H}_{p q}^{\alpha, \frac{1}{2} \alpha}\left(\mathbb{R}^{n} \times(0, T)\right)} \leq c\left\|\tilde{f}_{i}-\tilde{f}_{i}^{*}\right\|_{\dot{H}_{p q, 0}^{\alpha-2, \frac{1}{2} \alpha-1}\left(\mathbb{R}^{n} \times(0, T)\right)} \\
\leq c\left\|\tilde{f}_{i}\right\|_{\dot{H}_{p q, 0}^{\alpha-2, \frac{1}{2} \alpha-1}\left(\mathbb{R}^{n} \times(0, T)\right)} \leq c\left\|f_{i}\right\|_{\dot{H}_{p q, 0}^{\alpha-2, \frac{1}{2} \alpha-1}\left(\mathbb{R}_{+}^{n} \times(0, T)\right)} .
\end{gathered}
$$

This completes the proof.

Lemma 3.2. Let the assumption in Lemma 3.1 hold. If $w$ is given in (3.12), then for $1 \leq i \leq n$

$$
\left\|\frac{\partial}{\partial x_{i}} w\right\|_{\dot{H}_{p q}^{\alpha, \frac{1}{2} \alpha}\left(\mathbb{R}_{+}^{n} \times(0, T)\right)} \leq c \sum_{1 \leq j \leq n-1}\left\|f_{j}\right\|_{\dot{H}_{p q, 0}^{\alpha-2, \frac{1}{2} \alpha-1}\left(\mathbb{R}_{+}^{n} \times(0, T)\right)} .
$$

Proof. We note that $w=0$ on $\left\{x_{n}=0\right\}$ and $w$ solves for each $t$

$$
\Delta w(x, t)=-\frac{1}{2} \sum_{1 \leq j \leq n-1} \frac{\partial}{\partial x_{j}} \mathcal{U}^{*} f_{j}(x, t)+\frac{\partial}{\partial x_{n}} \mathcal{I} \sum_{1 \leq j \leq n-1} \frac{\partial}{\partial x_{j}} \mathcal{U}^{*} f_{j}(x, t) \quad \text { in } \mathbb{R}_{+}^{n} .
$$

We denote by $R^{\prime}=\left(R_{1}^{\prime}, \cdots, R_{n-1}^{\prime}\right)$ the Riesz transforms in $\mathbb{R}^{n-1}$ and we set

$$
\begin{gathered}
F_{j}:=-\frac{1}{2} \mathcal{U}^{*} f_{j}, \quad j=1 \cdots, n-1, \\
F_{n}:=\mathcal{I} \sum_{1 \leq j \leq n-1} \frac{\partial}{\partial x_{j}} \mathcal{U}^{*} f_{j}(x, t)=\sum_{1 \leq j \leq n-1} R_{j}^{\prime} \mathcal{U}^{*} f_{j}\left(x^{\prime}, x_{n}, t\right) .
\end{gathered}
$$

Then, the right-hand side of (3.16) is equal to div F. By the representation formula of Poisson problem for Lapalce equation in $\mathbb{R}_{+}^{n}$, we have

$$
\begin{aligned}
w(x, t) & =-\int_{\mathbb{R}_{+}^{n}}\left(E(x-y)-E\left(x-y^{*}\right)\right) \operatorname{div} F(y, t) d y \\
& =\int_{\mathbb{R}_{+}^{n}} D_{y}\left(E(x-y)-E\left(x-y^{*}\right)\right) \cdot F(y, t) d y .
\end{aligned}
$$

Next, we show that for $k=1,2,3$

$$
\int_{0}^{T}\left\|D_{x}^{k} w(\cdot, t)\right\|_{L^{p}\left(\mathbb{R}_{+}^{n}\right)}^{q} d t \leq c \sum_{1 \leq j \leq n-1} \int_{0}^{T}\left\|\mathcal{U}^{*} f_{j}(\cdot, t)\right\|_{\dot{H}_{p}^{k-1}\left(\mathbb{R}_{+}^{n}\right)}^{q} d t .
$$

Indeed, in case that $k=1,2$, we observe that

$$
\int_{0}^{T}\left\|D_{x}^{k} w(\cdot, t)\right\|_{L^{p}\left(\mathbb{R}_{+}^{n}\right)}^{q} d t \leq c \int_{0}^{T} \sum_{1 \leq j \leq n}\left\|\nabla^{k-1} F_{j}(\cdot, t)\right\|_{L^{p}\left(\mathbb{R}_{+}^{n}\right)}^{q}
$$




$$
\leq c \sum_{1 \leq j \leq n-1} \int_{0}^{T}\left\|D_{x}^{k-1} \mathcal{U}^{*} f_{j}(\cdot, t)\right\|_{L^{p}\left(\mathbb{R}_{+}^{n}\right)}^{q} d t \leq c \sum_{1 \leq j \leq n-1} \int_{0}^{T}\left\|\mathcal{U}^{*} f_{j}(\cdot, t)\right\|_{\dot{H}_{p}^{k-1}\left(\mathbb{R}_{+}^{n}\right)}^{q} d t .
$$

When $k=3$ and $(l, m, i) \neq(n, n, n)$, we have

$$
\begin{gathered}
\int_{0}^{T}\left\|D_{l m i} w(\cdot, t)\right\|_{L^{p}\left(\mathbb{R}_{+}^{n}\right)}^{q} d t \leq c \int_{0}^{T} \sum_{1 \leq j \leq n}\left\|D_{x}^{2} F_{j}(\cdot, t)\right\|_{L^{p}\left(\mathbb{R}_{+}^{n}\right)}^{q} \\
\leq c \sum_{1 \leq j \leq n-1} \int_{0}^{T}\left\|D_{x}^{2} \mathcal{U}^{*} f_{j}(\cdot, t)\right\|_{L^{p}\left(\mathbb{R}_{+}^{n}\right)}^{q} d t \leq c \sum_{1 \leq j \leq n-1} \int_{0}^{T}\left\|\mathcal{U}^{*} f_{j}(\cdot, t)\right\|_{\dot{H}_{p}^{2}\left(\mathbb{R}_{+}^{n}\right)}^{q} d t .
\end{gathered}
$$

It remains to estimate the case that $(l, m, i)=(n, n, n)$. We note first that

$$
\begin{aligned}
D_{n n n} w(x, t)= & D_{n n} \int_{\mathbb{R}_{+}^{n}} D_{y_{n}}\left(E(x-y)+E\left(x-y^{*}\right)\right) \operatorname{div} F(y, t) d y \\
= & -D_{n n} \int_{\mathbb{R}_{+}^{n}}\left(E(x-y)+E\left(x-y^{*}\right)\right) D_{y_{n}} \operatorname{div} F(y, t) d y \\
& +D_{n n} \int_{\mathbb{R}^{n-1}} E\left(x^{\prime}-y^{\prime}, x_{n}\right) \operatorname{div} F\left(y^{\prime}, 0, t\right) d y^{\prime}:=J_{1}+J_{2} .
\end{aligned}
$$

As in the above cases, we estimate the term $J_{1}$ as follows:

$$
\int_{0}^{T}\left\|J_{1}(\cdot, t)\right\|_{L^{p}\left(\mathbb{R}_{+}^{n}\right)}^{q} d t \leq \int_{0}^{T}\left\|D_{y_{n}} \operatorname{div} F(\cdot, t)\right\|_{L^{p}\left(\mathbb{R}_{+}^{n}\right)}^{q} d t \leq c \sum_{1 \leq j \leq n-1} \int_{0}^{T}\left\|\mathcal{U}^{*} f_{j}(\cdot, t)\right\|_{\dot{H}_{p}^{2}\left(\mathbb{R}_{+}^{n}\right)}^{q} d t .
$$

On the other hand, we rewrite $J_{2}$ as

$$
J_{2}(x, t)=D_{n} \int_{\mathbb{R}^{n-1}} P_{x_{n}}\left(x^{\prime}-y^{\prime}\right) \operatorname{div} F\left(y^{\prime}, 0, t\right) d y^{\prime},
$$

where $P_{x_{n}}$ is the Poisson Kernel in a half-space. With the aid of estimates in proposition 2.3 ,

$$
\begin{gathered}
\int_{0}^{T}\left\|J_{2}(\cdot, t)\right\|_{L^{p}\left(\mathbb{R}_{+}^{n}\right)}^{q} d t \leq \int_{0}^{T}\|\operatorname{div} F(\cdot, 0, t)\|_{\dot{B}_{p}^{1-\frac{1}{p}}}^{q} d t \leq \int_{0}^{T}\|F(\cdot, 0, t)\|_{\dot{B}_{p}^{2-\frac{1}{p}}\left(\mathbb{R}^{n-1}\right)}^{q} d t \\
\leq \int_{0}^{T}\|F(\cdot, t)\|_{\dot{H}_{p}^{2}\left(\mathbb{R}_{+}^{n}\right)}^{q} d t \leq c \sum_{1 \leq j \leq n-1} \int_{0}^{T}\left\|\mathcal{U}^{*} f_{j}(\cdot, t)\right\|_{\dot{H}_{p}^{2}\left(\mathbb{R}_{+}^{n}\right)}^{q} d t .
\end{gathered}
$$

Summing up (3.19)-(3.22), we obtain (3.18). Using (3.18) and Proposition2.2 we get for $i=1, \cdots, n$

$$
\begin{gathered}
\int_{0}^{T}\left\|\frac{\partial}{\partial x_{i}} w(\cdot, t)\right\|_{\dot{H}_{p}^{\alpha}\left(\mathbb{R}_{+}^{n}\right)}^{q} d t \leq c \sum_{1 \leq j \leq n-1} \int_{0}^{T}\left\|\mathcal{U}^{*} f_{j}(\cdot, t)\right\|_{\dot{H}_{p}^{\alpha}\left(\mathbb{R}_{+}^{n}\right)}^{q} d t \\
\leq c \sum_{1 \leq j \leq n-1}\left\|f_{j}\right\|_{\dot{H}_{p q, 0}^{\alpha-2, \frac{1}{2} \alpha-1}}^{q}\left(\mathbb{R}_{+}^{n} \times(0, T)\right)
\end{gathered}
$$

For the time regularity of $w$, we have

$$
D_{t}^{\frac{\alpha}{2}} w(x, t)=\int_{0}^{x_{n}} \int_{\mathbb{R}^{n-1}} E(x-y) \sum_{1 \leq j \leq n-1} \frac{\partial}{\partial y_{j}} D_{t}^{\frac{\alpha}{2}} \mathcal{U}^{*} f_{j}(y, t) d y .
$$


Applying the above argument, for $1 \leq i \leq n$ we get

$$
\begin{aligned}
\int_{0}^{T}\left\|D_{t}^{\frac{\alpha}{2}} \frac{\partial}{\partial x_{i}} w\right\|_{L^{p}\left(\mathbb{R}_{+}^{n}\right)}^{q} d t & \leq c \sum_{1 \leq j \leq n-1} \int_{0}^{T}\left\|D_{t}^{\frac{\alpha}{2}} \mathcal{U}^{*} f_{j}(x, \cdot)\right\|_{L^{p}\left(\mathbb{R}_{+}^{n}\right)}^{q} d x \\
& \leq c \sum_{1 \leq j \leq n-1}\left\|f_{j}\right\|_{\dot{H}_{p q, 0}^{\alpha-2, \frac{1}{2} \alpha-1}}^{q} .
\end{aligned}
$$

Combining (3.23) and (3.24), we obtain (3.15). This completes the proof.

Lemma 3.3. Let the assumption in Lemma 3.1 hold. If $\mathcal{U}^{*} f_{j}$ is given in (3.13), then

$$
\left\|\mathcal{I} \sum_{1 \leq j \leq n-1} \frac{\partial}{\partial x_{j}} \mathcal{U}^{*} f_{j}(\cdot, t)\right\|_{\dot{H}_{p q}^{\alpha, \frac{1}{2} \alpha}\left(\mathbb{R}_{+}^{n} \times I\right)} \leq c \sum_{1 \leq j \leq n-1}\left\|f_{j}\right\|_{\dot{H}_{p q, 0}^{\alpha-2, \frac{1}{2} \alpha-1}\left(\mathbb{R}_{+}^{n} \times I\right)} .
$$

Proof. Since for $1 \leq j \leq n-1, D_{x}^{2} \mathcal{I} \frac{\partial}{\partial x_{j}} \mathcal{U}^{*} f_{j}(x, t)=\mathcal{I} \frac{\partial}{\partial x_{j}} D_{x}^{2} \mathcal{U}^{*} f_{j}(x, t)$, recalling (3.17), we have

$$
\begin{array}{r}
\int_{0}^{T}\left\|D_{x}^{2} \mathcal{I} \sum_{1 \leq j \leq n-1} \frac{\partial}{\partial x_{j}} \mathcal{U}^{*} f_{j}(\cdot, t)\right\|_{L^{p}\left(\mathbb{R}_{+}^{n}\right)}^{q} d t \leq c \sum_{1 \leq j \leq n-1} \int_{0}^{T}\left\|D_{x}^{2} \mathcal{U}^{*} f_{j}(\cdot, t)\right\|_{L^{p}\left(\mathbb{R}_{+}^{n}\right)}^{q} d t \\
\int_{0}^{T}\left\|\mathcal{I} \sum_{1 \leq j \leq n-1} \frac{\partial}{\partial x_{j}} \mathcal{U}^{*} f_{j}(\cdot, t)\right\|_{L^{p}\left(\mathbb{R}_{+}^{n}\right)}^{q} d t \leq c \sum_{1 \leq j \leq n-1} \int_{0}^{T}\left\|\mathcal{U}^{*} f_{j}(\cdot, t)\right\|_{L^{p}\left(\mathbb{R}_{+}^{n}\right)}^{q} d t .
\end{array}
$$

Via (3.26), (3.27), Proposition 2.2 and Lemma 3.1, we obtain

$$
\begin{aligned}
\int_{0}^{T}\left\|\mathcal{I} \sum_{1 \leq j \leq n-1} \frac{\partial}{\partial x_{j}} \mathcal{U}^{*} f_{j}(\cdot, t)\right\|_{\dot{H}_{p}^{\alpha}\left(\mathbb{R}_{+}^{n}\right)}^{q} d t & \leq c \sum_{1 \leq j \leq n-1} \int_{0}^{T}\left\|\mathcal{U}^{*} f_{j}(\cdot, t)\right\|_{\dot{H}_{p}^{\alpha}\left(\mathbb{R}_{+}^{n}\right)}^{q} d t \\
& \leq c \sum_{1 \leq j \leq n-1}\left\|f_{j}\right\|_{\dot{H}_{p q, 0}^{\alpha-2, \frac{1}{2} \alpha-1}}^{q}\left(\mathbb{R}_{+}^{n} \times(0, T)\right)
\end{aligned}
$$

For the time regularity, note that $1 \leq j \leq n-1, D_{t}^{\frac{\alpha}{2}} \mathcal{I} \frac{\partial}{\partial x_{j}} \mathcal{U}^{*} f_{j}(x, t)=\mathcal{I} \frac{\partial}{\partial x_{j}} D_{t}^{\frac{\alpha}{2}} \mathcal{U}^{*} f_{j}(x, t)$, recalling (3.17) and Lemma 3.1 we have

$$
\begin{aligned}
\int_{0}^{T}\left\|D_{t}^{\frac{\alpha}{2}} \mathcal{I} \sum_{1 \leq j \leq n-1} \frac{\partial}{\partial x_{j}} \mathcal{U}^{*} f_{j}(\cdot, t)\right\|_{L^{p}\left(\mathbb{R}_{+}^{n}\right)}^{q} d t & \leq c \sum_{1 \leq j \leq n-1} \int_{0}^{T}\left\|D_{t}^{\frac{\alpha}{2}} \mathcal{U}^{*} f_{j}(\cdot, t)\right\|_{L^{p}\left(\mathbb{R}_{+}^{n}\right)}^{q} d t \\
& \left.\leq c \sum_{1 \leq j \leq n-1}\left\|f_{j}\right\|_{\dot{H}_{p q, 0}^{\alpha-2, \frac{1}{2} \alpha-1}}^{q} \mathbb{R}_{+}^{n} \times(0, T)\right)
\end{aligned}
$$

This completes the proof.

Therefore, with the aid of Lemma 3.1. Lemma 3.2 and Lemma 3.3. we conclude that

$$
\|v\|_{\dot{H}_{p q}^{\alpha, \frac{1}{2} \alpha}\left(\mathbb{R}_{+}^{n} \times(0, T)\right)} \leq c\|f\|_{\dot{H}_{p q, 0}^{\alpha-2, \frac{1}{2} \alpha-1}\left(\mathbb{R}_{+}^{n} \times(0, T)\right)} .
$$

Following similar procedure, we can show that

$$
\|v\|_{H_{p q}^{\alpha, \frac{1}{2} \alpha}\left(\mathbb{R}_{+}^{n} \times(0, T)\right)} \leq c\|f\|_{H_{p q, 0}^{\alpha-2, \frac{1}{2} \alpha-1}\left(\mathbb{R}_{+}^{n} \times(0, T)\right)} .
$$

Since its verification of (3.33) is almost the same, we omit the detail. 


\subsection{Estimates of pressure}

We estimate the pressure term. Recalling the formula (3.4)-(3.6), we split $p$ in two terms, i.e. $p(x, t)=p_{1}(x, t)+p_{2}(x, t)$, where

$$
\begin{aligned}
& p_{1}(x, t)=4 \sum_{1 \leq j \leq n-1} \int_{\mathbb{R}^{n-1}} \frac{\partial^{2} E\left(x^{\prime}-z^{\prime}, x_{n}\right)}{\partial x_{j} \partial x_{n}} \int_{0}^{t} \int_{\mathbb{R}_{+}^{n}} \Gamma\left(z^{\prime}-y^{\prime}, y_{n}, t-\tau\right) f_{j}(y, \tau) d y d \tau d z^{\prime}, \\
& p_{2}(x, t)=4 \sum_{1 \leq j \leq n-1} \int_{\mathbb{R}^{n-1}} \frac{\partial E\left(x^{\prime}-z^{\prime}, x_{n}\right)}{\partial x_{j}} \int_{0}^{t} \int_{\mathbb{R}_{+}^{n}} \frac{\partial \Gamma\left(z^{\prime}-y^{\prime}, y_{n}, t-\tau\right)}{\partial y_{n}} f_{j}(y, \tau) d y d \tau d z^{\prime} .
\end{aligned}
$$

Lemma 3.4. Let the assumption in Lemma 3.1 hold. If $p_{1}$ is given in (3.34), then

$$
\left\|p_{1}\right\|_{L^{q}\left((0, T) ; \dot{H}_{p}^{\alpha-1}\left(\mathbb{R}_{+}^{n}\right)\right)} \leq c\|f\|_{\dot{H}_{p q, 0}^{\alpha-2, \frac{1}{2} \alpha-1}\left(\mathbb{R}_{+}^{n} \times I\right)} .
$$

Proof. We note that

$$
p_{1}(x, t)=4 \sum_{1 \leq j \leq n-1} P_{x_{n}} \frac{\partial}{\partial x_{j}} U f_{j}\left(x^{\prime}, t\right),
$$

where $P_{x_{n}}$ is the Poisson integral of the Laplace equation and

$$
U f_{j}\left(y^{\prime}, t\right)=\int_{0}^{t} \int_{\mathbb{R}_{+}^{n}} \Gamma\left(z^{\prime}-y^{\prime}, z_{n}, t\right) f_{j}(z, \tau) d z d \tau
$$

Hence, by the properties of Poisson integral of Laplace equation (see Proposition 2.3), for $\alpha \geq 1$,

$$
\begin{aligned}
\int_{0}^{T}\left\|p_{1}(\cdot, t)\right\|_{\dot{H}_{p}^{\alpha-1}\left(\mathbb{R}_{+}^{n}\right)}^{q} d t & \leq c \sum_{1 \leq j \leq n-1} \int_{0}^{T}\left\|\frac{\partial}{\partial x_{j}} U f_{j}(\cdot, t)\right\|_{\dot{B}_{p}^{\alpha-1-\frac{1}{p}}\left(\mathbb{R}^{n-1}\right)}^{q} d t \\
& \leq c \sum_{1 \leq j \leq n-1} \int_{0}^{T}\left\|U f_{j}(\cdot, t)\right\|_{\dot{B}_{p}^{\alpha-\frac{1}{p}}}^{q} d t .
\end{aligned}
$$

Let $\mathcal{U} f_{j}(y, t)=\int_{0}^{t} \int_{\mathbb{R}_{+}^{n}} \Gamma\left(z^{\prime}-y^{\prime}, z_{n}-y_{n}, t\right) f_{j}(z, \tau) d z d \tau$ such that $\left.\mathcal{U} f_{j}\right|_{y_{n}=0}=U f_{j}$. Using Proposition 2.3 and Proposition [2.2, we obtain

$$
\begin{gathered}
\left.\int_{0}^{T}\left\|p_{1}\right\|_{\dot{H}_{p}^{\alpha-1}\left(\mathbb{R}_{+}^{n}\right)}^{q} d t \leq \sum_{1 \leq j \leq n-1} \int_{0}^{T}\left\|U f_{j}\right\|_{\dot{B}_{p}^{\alpha-\frac{1}{p}}}^{q} d t \leq c \sum_{1 \leq j \leq n-1} \int_{0}^{T}\left\|\mathcal{U} f_{j}\right\|_{\left.\dot{H}_{p}^{\alpha-1}\right)}^{q} d \mathbb{R}_{+}^{n}\right) \\
\leq c \sum_{1 \leq j \leq n-1}\left\|f_{j}\right\|_{\dot{H}_{p q, 0}^{\alpha-2, \frac{1}{2} \alpha-1}}^{q} d t \\
\left.\left(\mathbb{R}_{+}^{n} \times I\right)\right)
\end{gathered}
$$

This completes the proof.

Lemma 3.5. Let the assumption in Lemma 3.1 hold. If $p_{2}$ is given in (3.35), then for $\alpha>1+\frac{1}{p}$,

$$
\left\|p_{2}\right\|_{L^{q}\left((0, T) ; \dot{H}_{p}^{\alpha-1}\left(\mathbb{R}_{+}^{n}\right)\right)} \leq c \sum_{1 \leq j \leq n-1}\left\|f_{j}\right\|_{\dot{H}_{p q, 0}^{\alpha-2, \frac{1}{2} \alpha-1}\left(\mathbb{R}_{+}^{n} \times I\right)}
$$


Proof. As before, we denote by $P_{x_{n}}$ the Poisson integral of the Laplace equation in $\mathbb{R}_{+}^{n}$ and $R_{j}^{\prime}$ indicates Riesz transform in $\mathbb{R}^{n-1}$. We then rewrite $p_{2}$ as

$$
p_{2}(x, t)=4 \sum_{1 \leq j \leq n-1} P_{x_{n}} R_{j}^{\prime} U_{2} f_{j}\left(x^{\prime}, t\right),
$$

where

$$
U_{2} f_{j}\left(y^{\prime}, t\right)=\int_{0}^{t} \int_{\mathbb{R}_{+}^{n}} \frac{\partial}{\partial z_{n}} \Gamma\left(z^{\prime}-y^{\prime}, z_{n}, t\right) f_{j}(z, \tau) d z d \tau .
$$

We note that $U_{2} f_{j}=\left.\mathcal{U}_{2} f_{j}\right|_{y_{n}=0}$, where

$$
\mathcal{U}_{2} f_{j}(y, t)=-\frac{\partial}{\partial y_{n}} \mathcal{U} f_{j}(y, t)=\int_{0}^{t} \int_{\mathbb{R}_{+}^{n}} \frac{\partial}{\partial z_{n}} \Gamma\left(z^{\prime}-y^{\prime}, z_{n}-y_{n}, t\right) f_{j}(z, \tau) d z d \tau .
$$

Via the properties of Poisson integral and Proposition 2.3, for $\alpha>1+\frac{1}{p}$ we have

$$
\begin{gathered}
\int_{0}^{T}\left\|p_{2}(\cdot, t)\right\|_{\dot{H}_{p}^{\alpha-1}\left(\mathbb{R}_{+}^{n}\right)}^{q} d t \leq c \sum_{1 \leq j \leq n-1} \int_{0}^{T}\left\|U_{2} f_{j}(\cdot, t)\right\|_{\dot{B}_{p}^{\alpha-1-\frac{1}{p}}}^{q} d t \\
\leq c \sum_{1 \leq j \leq n-1}\left\|f_{j}\right\|_{\left.\dot{H}_{p q, 0}^{\alpha-2,1}\right)} d t{ }_{\left(\mathbb{R}_{+}^{n} \times I\right)}
\end{gathered}
$$

This completes the proof.

Remark In case $1 \leq \alpha \leq 1+\frac{1}{p}$, the pressure $p_{2}$ is decomposed to $p_{2}=Q+\partial_{t} P$ such that

$$
\begin{gathered}
\|Q\|_{L^{q}\left((0, T) ; \dot{H}_{p}^{\alpha-1}\left(\mathbb{R}_{+}^{n}\right)\right)} \leq c\|f\|_{\dot{H}_{p q, 0}^{\alpha-2, \frac{1}{2} \alpha-1}\left(\mathbb{R}_{+}^{n} \times I\right)}, \\
\|P\|_{L^{q}\left(0, T ; \dot{H}_{p}^{\alpha+1}\left(\mathbb{R}_{+}^{n}\right)\right)} \leq c\|f\|_{\left.\dot{H}_{p}^{\alpha-2, \frac{1}{2} \alpha-1}\left(\mathbb{R}_{+}^{n} \times I\right)\right)} .
\end{gathered}
$$

Indeed, since $\operatorname{div} f=0$, we get

$$
\begin{aligned}
p_{2}(x, t) & =4 \sum_{1 \leq j \leq n-1} \int_{\mathbb{R}^{n-1}} \frac{\partial E\left(x^{\prime}-z^{\prime}, x_{n}\right)}{\partial x_{j}} \int_{0}^{t} \int_{\mathbb{R}_{+}^{n}} \frac{\partial \Gamma\left(z^{\prime}-y^{\prime}, y_{n}, t-\tau\right)}{\partial y_{n}} f_{j}(y, \tau) d y d \tau d z^{\prime} . \\
& =4 \int_{\mathbb{R}^{n-1}} E\left(x^{\prime}-z^{\prime}, x_{n}\right) \int_{0}^{t} \int_{\mathbb{R}_{+}^{n}} \frac{\partial \Gamma\left(z^{\prime}-y^{\prime}, y_{n}, t-\tau\right)}{\partial y_{n}} D_{y_{n}} f_{n}(y, \tau) d y d \tau d z^{\prime} \\
& =4 \int_{\mathbb{R}^{n-1}} E\left(x^{\prime}-z^{\prime}, x_{n}\right) \int_{0}^{t} \int_{\mathbb{R}_{+}^{n}} \Delta^{\prime} \Gamma\left(z^{\prime}-y^{\prime}, y_{n}, t-\tau\right) f_{n}(y, \tau) d y d \tau d z^{\prime} \\
& +4 D_{t} \int_{\mathbb{R}^{n-1}} E\left(x^{\prime}-z^{\prime}, x_{n}\right) \int_{0}^{t} \int_{\mathbb{R}_{+}^{n}} \Gamma\left(z^{\prime}-y^{\prime}, y_{n}, t-\tau\right) f_{n}(y, \tau) d y d \tau d z^{\prime} \\
& :=Q+\partial_{t} P .
\end{aligned}
$$

The estimate (3.41) is similar to the lemma 3.4 and we skip its details. For the second term $P$, we estimate

$$
\begin{gathered}
\|P\|_{L^{q}\left(0, T ; \dot{H}_{p}^{\alpha+1}\left(\mathbb{R}_{+}^{n}\right)\right)} \leq c\left\|\left.P\right|_{x_{n}=0}\right\|_{L^{q}\left(0, T ; \dot{B}_{p}^{\alpha+1-\frac{1}{p}}\left(\mathbb{R}^{n-1}\right)\right)} \leq c\left\|U f_{n}\right\|_{L^{q}\left(0, T ; \dot{B}_{p}^{\alpha-\frac{1}{p}}\left(\mathbb{R}^{n-1}\right)\right)} \\
\leq c\left\|\mathcal{U} f_{n}\right\|_{L^{q}\left(0, T ; \dot{H}_{p}^{\alpha}\left(\mathbb{R}_{+}^{n}\right)\right)} \leq c\left\|f_{n}\right\|_{\left.\dot{H}_{p}^{\alpha-2, \frac{1}{2} \alpha-1}\left(\mathbb{R}_{+}^{n} \times I\right)\right)} .
\end{gathered}
$$

However, the estimate (3.44) of $\partial_{t} P$ is not available and thus neither is the pressure $p$. This is what was essentially shown in [17, Theorem 1.5] for the case $\alpha=1$. Our estimates of the pressure above, however, implies that if $f$ has a bit better regularity, i.e. $f \in \dot{H}_{p q, 0}^{\alpha-2, \frac{1}{2} \alpha-1}\left(\mathbb{R}_{+}^{n} \times I\right)$ with $\alpha>1+\frac{1}{p}$, the pressure $p$ can be controlled in terms of $f$. 


\section{Stokes system with $f=0$ and $v_{0} \neq 0$}

In this section, we consider the Stokes system with homogeneous external force and non-zero initial data in $\mathbb{R}_{+}^{n} \times(0, \infty)$, namely

$$
\begin{gathered}
v_{t}-\nu \Delta v+\nabla p=0, \quad \operatorname{div} v=0 \quad \text { in } \quad Q_{T}^{+}:=\mathbb{R}_{+}^{n} \times[0, T), \\
v(x, 0)=v_{0} \quad \text { and } \quad v(x, t)=0, \quad x \in \partial \mathbb{R}_{+}^{n}=\mathbb{R}^{n-1} .
\end{gathered}
$$

We review a solution representation of (4.1)-(4.2) formulated by S. Ukai (see 32]). Let $R=\left(R^{\prime}, R_{n}\right)$ and $S=\left(S_{1}, \cdots, S_{n-1}\right)$ be Riesz's operators in $\mathbb{R}^{n}$ and $\mathbb{R}^{n-1}$, respectively, that is,

$$
\begin{aligned}
R_{i} f(x) & =c_{n} \int_{\mathbb{R}^{n}} \frac{x_{i}-y_{i}}{|x-y|^{n+1}} f(y) d y, \quad x \in \mathbb{R}^{n}, \quad 1 \leq i \leq n, \\
S_{i} f\left(x^{\prime}, x_{n}\right) & =c_{n-1} \int_{\mathbb{R}^{n-1}} \frac{x_{i}-y_{i}}{\left|x^{\prime}-y^{\prime}\right|^{n}} f\left(y^{\prime}, x_{n}\right) d y^{\prime}, \quad x^{\prime} \in \mathbb{R}^{n-1}, \quad 1 \leq i \leq n-1 .
\end{aligned}
$$

The functional operator $V_{1}$ and $V_{2}$ are defined by

$$
V_{1} v_{0}(x)=-\left(S \cdot v_{0}\left(\cdot, x_{n}\right)\right)\left(x^{\prime}\right)+v_{0}^{n}(x), \quad V_{2} u_{0}(x)=v_{0}^{\prime}(x)+\left(S v_{0}^{n}\left(\cdot, x_{n}\right)\right)\left(x^{\prime}\right) .
$$

Further, let $\gamma$ be the restriction operator from $\mathbb{R}_{+}^{n}$ to $\mathbb{R}^{n-1}$, namely $\gamma g=\left.g\right|_{\mathbb{R}^{n-1}}$. For a given function $g: \mathbb{R}_{+}^{n} \rightarrow \mathbb{R}$ we define a functional operator $U$ by

$$
U g(x)=r R^{\prime} \cdot S\left(R^{\prime} \cdot S+R_{n}\right) \operatorname{eg}(x), \quad x \in \mathbb{R}_{+}^{n},
$$

where $r$ be the restriction operator from $\mathbb{R}^{n}$ to $\mathbb{R}_{+}^{n}$ and $e$ the zero extension operator from $\mathbb{R}_{+}^{n}$ over $\mathbb{R}^{n}$, i.e.

$$
r f=\left.f\right|_{\mathbb{R}_{+}^{n}}, \quad e f= \begin{cases}f, & \text { for } \quad x_{n}>0 \\ 0, & \text { for } \quad x_{n}<0 .\end{cases}
$$

We also define the integral operators $D$ and $E(t)$ by

$$
\begin{gathered}
D g(x)=c_{n} \int_{\mathbb{R}^{n-1}} \frac{x_{n}}{\left(\left|x^{\prime}-y^{\prime}\right|^{2}+x_{n}^{2}\right)^{\frac{n}{2}}} g\left(y^{\prime}\right) d y^{\prime}, \\
E(t) g(x)=\int_{\mathbb{R}_{+}^{n}}\left(\Gamma(x-y, t)-\Gamma\left(x-y^{*}, t\right)\right) g(y) d y .
\end{gathered}
$$

Then, the solution of (4.1)-(4.2) is represented by

$$
\begin{gathered}
v^{\prime}(x, t)=E(t) V_{2} u_{0}-S U r E(t) e V_{1} u_{0}, \quad v^{n}(x, t)=U r E(t) e V_{1} u_{0}(x, t), \\
p(x, t)=-D \gamma \partial_{n} E(t) e V_{1} u_{0} .
\end{gathered}
$$

We denote, for simplicity, by $X$ one of function spaces $H_{p}^{\beta}\left(\mathbb{R}^{n}\right), \dot{H}_{p}^{\beta}\left(\mathbb{R}^{n}\right), B_{p}^{\beta}\left(\mathbb{R}^{n}\right)$ and $\dot{B}_{p}^{\beta}\left(\mathbb{R}^{n}\right)$. We then note that the following functionals are bounded operators:

$$
R_{i}: X \rightarrow X, i=1, \cdots, n, \quad S_{i}: X \rightarrow X, i=1, \cdots, n-1 .
$$

Similarly we mean by $Y_{+}$one of $H_{p, 0}^{\beta}\left(\mathbb{R}_{+}^{n}\right), B_{p, 0}^{\beta}\left(\mathbb{R}_{+}^{n}\right), \dot{H}_{p, 0}^{\beta}\left(\mathbb{R}_{+}^{n}\right)$ and $\dot{B}_{p, 0}^{\beta}\left(\mathbb{R}_{+}^{n}\right)$. We also observe that

$$
V_{1}: Y_{+} \rightarrow Y_{+}, \quad V_{2}: Y_{+} \rightarrow Y_{+}, \quad U: Y_{+} \rightarrow Y_{+}
$$

are are bounded operators, where we used that $e: B_{q, 0}^{\alpha}\left(\mathbb{R}_{+}^{n}\right) \rightarrow B_{q}^{\alpha}\left(\mathbb{R}^{n}\right)$ or $e: \dot{B}_{q, 0}^{\alpha}\left(\mathbb{R}_{+}^{n}\right) \rightarrow \dot{B}_{q}^{\alpha}\left(\mathbb{R}^{n}\right)$ is a bounded operator.

In next proposition we consider the heat equation with an initial data in Besov spaces in $\mathbb{R}^{n}$. 
Proposition 4.1. Let $\alpha \geq 0$. Suppose that $u_{0} \in \dot{B}_{p q}^{\alpha-\frac{2}{q}}\left(\mathbb{R}^{n}\right)$. For each $(x, t) \in \mathbb{R}^{n} \times(0, \infty)$ we define

$$
u(x, t):= \begin{cases}<u_{0}(\cdot), \Gamma(x-\cdot, t)>, & \text { if } 0 \leq \alpha<\frac{2}{q}, \\ \int_{\mathbb{R}^{n}} \Gamma(x-y, t) u_{0}(y) d y, & \text { if } \frac{2}{q} \leq \alpha .\end{cases}
$$

Then $\left.u \in \dot{H}_{p q, *}^{\alpha, \frac{1}{2} \alpha}\left(\mathbb{R}^{n} \times(0, \infty)\right)\right)$ and the following estimate is satisfied:

$$
\|u\|_{\dot{H}_{p q, *}^{\alpha, \frac{1}{2} \alpha}\left(\mathbb{R}^{n} \times(0, \infty)\right)} \leq c\left\|u_{0}\right\|_{\dot{B}_{p q}^{\alpha-\frac{2}{q}}\left(\mathbb{R}^{n}\right)} .
$$

If $u_{0} \in B_{p q}^{\alpha-\frac{2}{q}}\left(\mathbb{R}^{n}\right)$, then $u \in H_{p q, *}^{\alpha, \frac{1}{2} \alpha}\left(\mathbb{R}^{n} \times(0, \infty)\right)$ and

$$
\|u\|_{H_{p q, *}^{\alpha, \frac{1}{2} \alpha}\left(\mathbb{R}^{n} \times(0, \infty)\right)} \leq c\left\|u_{0}\right\|_{B_{p q}^{\alpha-\frac{2}{q}}\left(\mathbb{R}^{n}\right)} .
$$

Although Proposition 4.1 may be known in experts, we are not able to find it in the literature and thus we provide the proof of our own. We start with next lemma related to theory of multipliers.

Lemma 4.2. Let $\Phi(\xi)=\hat{\phi}\left(2^{-1} \xi\right)+\hat{\phi}(\xi)+\hat{\phi}(2 \xi)$ with $\phi$ in the definition of Besov spaces. Suppose that $\Phi_{j}(\xi)=\Phi\left(2^{-j} \xi\right)$ and $\rho_{t j}(\xi)=\Phi_{j}(\xi) e^{-t|\xi|^{2}}$ for each integer $j$. Then, $\rho_{t j}(\xi)$ is a $L^{p}\left(\mathbb{R}^{n}\right)$-multiplier with the finite norm $M(t, j)$ for $1<p<\infty$ such that for $t>0$

$$
M(t, j) \leq c e^{-\frac{1}{4} t 2^{2 j}} \sum_{0 \leq i \leq n} t^{i} 2^{2 i j} \leq c e^{-\frac{1}{8} t 2^{2 j}} .
$$

Proof. We note that the $L^{p}\left(\mathbb{R}^{n}\right)$-multiplier norm $M(t, j)$ of $\rho_{t j}(\xi)$ is equal to the $L^{p}\left(\mathbb{R}^{n}\right)$-multiplier norm of $\rho_{t j}^{\prime}(\xi):=\Phi(\xi) e^{-t 2^{2 j}|\xi|^{2}}$ (see [2, Theorem 6.1.3]). Let $l$ be an integer with $1 \leq l \leq N$ and $\beta=\left(\beta_{1}, \beta_{2}, \cdots, \beta_{n}\right) \in \mathbb{R}^{n}$. Suppose $\left\{i_{1}, i_{2}, \cdots, i_{l}\right\} \subset\{1,2, \cdots, n\}$ and assume $\beta_{i_{1}}=\beta_{i_{2}}=\cdots=$ $\beta_{i_{l}}=1$ and $\beta_{i}=0$ for $i \neq i_{k}$ with $k=1, \cdots, l$. Since $\operatorname{supp}(\Phi) \subset\left\{\xi \in \mathbb{R}^{n}\left|\frac{1}{4}<\right| \xi \mid<4\right\}$, we have

$$
\left|D_{\xi}^{\beta} \rho_{t j}^{\prime}(\xi)\right| \lesssim e^{-\frac{1}{4} t 2^{2 j}} \chi_{\left\{\frac{1}{4}<|\xi|<4\right\}}(\xi) \sum_{0 \leq i \leq|\beta|} t^{i} 2^{2 i j}
$$

where $\chi_{A}$ is the characteristic function on a set $A$. Hence, for $A=\prod_{1 \leq i \leq l}\left[2^{k_{i}}, 2^{k_{i}+1}\right]$ we obtain

$$
\int_{A}\left|D_{\xi}^{\beta} \rho_{t j}^{\prime}(\xi)\right| d \xi_{\beta} \leq c e^{-\frac{1}{4} t 2^{2 j}} \sum_{0 \leq i \leq n} t^{i} 2^{2 i j}
$$

Due to Theorem $4.6^{\prime}$ in 29 , we deduce the lemma.

Note that if $g$ is smooth, then we have

$$
J_{1-\sigma} g(t)=\int_{t}^{\infty}(s-t)^{1-\sigma} g^{\prime}(s) d s, \quad D_{t}^{*} \sigma g(t)=\int_{t}^{\infty} \frac{g^{\prime}(s)}{(s-t)^{\sigma}} d s .
$$

Lemma 4.3. Suppose that $\Phi_{j}(\xi)=\Phi\left(2^{-j} \xi\right)$ and $\theta_{t j}(\xi)=\Phi_{j}(\xi)|\xi|^{2} \int_{t}^{\infty} \frac{e^{-s|\xi|^{2}}}{(s-t)^{\sigma}} d s$ for each integer $j$. Then, $\theta_{t j}(\xi)$ is a $L^{p}\left(\mathbb{R}^{n}\right)$-multiplier with the finite norm $N(t, j)$ for $1<p<\infty$ such that for $t>0$

$$
N(t, j) \leq c 2^{2 \sigma j} e^{-\frac{1}{4} t 2^{2 j}} \sum_{0 \leq i \leq n} t^{i} 2^{2 i j} \leq c 2^{2 \sigma j} e^{-\frac{1}{8} t 2^{2 j}} .
$$


Proof. We note that the $L^{p}\left(\mathbb{R}^{n}\right)$-multiplier norm $N(t, j)$ of $\theta_{t j}(\xi)$ is equal to the $L^{p}\left(\mathbb{R}^{n}\right)$-multiplier norm of $\theta_{t j}^{\prime}(\xi):=\Phi(\xi) 2^{2 j}|\xi|^{2} \int_{t}^{\infty} \frac{e^{-s 2^{2 j}|\xi|^{2}}}{(s-t)^{\sigma}} d s$ (see [2, Theorem 6.1.3]). Let $l$ be an integer with $1 \leq l \leq N$ and $\beta=\left(\beta_{1}, \beta_{2}, \cdots, \beta_{n}\right) \in \mathbb{R}^{n}$. Suppose $\left\{i_{1}, i_{2}, \cdots, i_{l}\right\} \subset\{1,2, \cdots, n\}$ and assume $\beta_{i_{1}}=$ $\beta_{i_{2}}=\cdots=\beta_{i_{l}}=1$ and $\beta_{i}=0$ for $i \neq i_{k}$ with $k=1, \cdots, l$. Since $\operatorname{supp}(\Phi) \subset\left\{\xi \in \mathbb{R}^{n}\left|\frac{1}{4}<\right| \xi \mid<4\right\}$, we have

$$
\begin{aligned}
\left|D_{\xi}^{\beta} \theta_{t j}^{\prime}(\xi)\right| & \lesssim 2^{2 j} \chi_{\left\{\frac{1}{4}<|\xi|<4\right\}}(\xi) \sum_{0 \leq i \leq|\beta|} \int_{t}^{\infty} \frac{\left(s 2^{2 j}\right)^{i} e^{-\frac{1}{4} s 2^{2 j}}}{(s-t)^{\sigma}} d s \\
& =2^{2 j} e^{-\frac{1}{4} t 2^{2 j}} \chi_{\left\{\frac{1}{4}<|\xi|<4\right\}}(\xi) \sum_{0 \leq i \leq|\beta|} 2^{2 i j} \int_{0}^{\infty} \frac{(s+t)^{i} e^{-\frac{1}{4} s 2^{2 j}}}{s^{\sigma}} d s \\
& =2^{2 j} e^{-\frac{1}{4} t 2^{2 j}} \chi_{\left\{\frac{1}{4}<|\xi|<4\right\}}(\xi) \sum_{0 \leq i \leq|\beta|} 2^{2 i j} \sum_{0 \leq l \leq i} t^{i-l} \int_{0}^{\infty} s^{l-\sigma} e^{-\frac{1}{4} s 2^{2 j}} d s \\
& =2^{2 j} e^{-\frac{1}{4} t 2^{2 j}} \chi_{\left\{\frac{1}{4}<|\xi|<4\right\}}(\xi) \sum_{0 \leq i \leq|\beta|} 2^{2 i j} \sum_{0 \leq l \leq i} t^{i-l} 2^{-2 j(l-\sigma+1)} \int_{0}^{\infty} s^{l-\sigma} e^{-\frac{1}{4} s} d s \\
& \lesssim 2^{2 \sigma j} e^{-\frac{1}{4} t 2^{2 j}} \chi_{\left\{\frac{1}{4}<|\xi|<4\right\}}(\xi) \sum_{0 \leq i \leq n} t^{i} 2^{2 i j} .
\end{aligned}
$$

Hence, for $A=\prod_{1 \leq i \leq l}\left[2^{k_{i}}, 2^{k_{i}+1}\right]$ we obtain

$$
\int_{A}\left|D_{\xi}^{\beta} \theta_{t j}^{\prime}(\xi)\right| d \xi_{\beta} \leq c 2^{2 \sigma j} e^{-\frac{1}{4} t 2^{2 j}} \sum_{0 \leq i \leq n} t^{i} 2^{2 i j} .
$$

Due to Theorem $4.6^{\prime}$ in [29], we deduce the lemma.

We are ready to give the proof of Proposition 4.1 .

Proof of Proposition 4.1 We only treat the case that $u_{0} \in \dot{B}^{k-\frac{2}{p}}\left(\mathbb{R}^{n}\right)$, since the proof of the case for $B^{k-\frac{2}{p}}\left(\mathbb{R}^{n}\right)$ is similar. We first show that for $k=0$

$$
\|v\|_{L_{x}^{p} L_{t}^{q}} \leq c\left\|u_{0}\right\|_{\dot{B}_{p q}^{-\frac{2}{q}}\left(\mathbb{R}^{n}\right)} .
$$

As usual, we may assume that $u_{0} \in C_{c}^{\infty}\left(\mathbb{R}^{n}\right)$, since $C_{c}^{\infty}\left(\mathbb{R}^{n}\right)$ is dense in $\dot{B}_{p q}^{-\frac{2}{q}}\left(\mathbb{R}^{n-1}\right)$. Using the dyadic partition of unity $\hat{\psi}(\xi)+\sum_{j=1}^{\infty} \hat{\phi}\left(2^{-j} \xi\right)=1$ for $\xi \in \mathbb{R}^{n-1}$, we can write

$$
\hat{v}(\xi, t)=\sum_{j=-\infty}^{\infty} \hat{\phi}\left(2^{-j} \xi\right) e^{-t|\xi|^{2}} \widehat{u_{0}}(\xi) .
$$

For $t>0$ we have

$$
\int_{\mathbb{R}^{n}}|v(x, t)|^{p} d x \leq \int_{\mathbb{R}^{n}}\left|\mathcal{F}^{-1}\left(\sum_{j=-\infty}^{\infty} e^{-t|\xi|^{2}} \hat{\phi}_{j}(\xi) \widehat{u_{0}}(\xi)\right)(x)\right|^{p} d x .
$$

We note that $\hat{\phi}_{j}=\Phi_{j} \hat{\phi}_{j}$ for all $j$, where $\Phi_{j}$ is defined in Lemma 4.2 and by Lemma 4.2, $\Phi_{j}(\xi) e^{-t|\xi|^{2}}$ is the $L^{p}\left(\mathbb{R}^{n}\right)$ - multipliers with the norms $M(t, j)$. Then we divide the sum as

$$
\begin{aligned}
& \int_{\mathbb{R}^{n}}\left|\mathcal{F}^{-1}\left(\sum_{j=-\infty}^{\infty} e^{-t|\xi|^{2}} \hat{\phi}_{j}(\xi) \widehat{u_{0}}(\xi)\right)(x)\right|^{p} d x=\int_{\mathbb{R}^{n}}\left|\mathcal{F}^{-1}\left(\sum_{j=-\infty}^{\infty} \Phi_{j}(\xi) e^{-t|\xi|^{2}} \hat{\phi}_{j}(\xi) \widehat{u_{0}}(\xi)\right)(x)\right|^{p} d x \\
& \leq\left(\sum_{2^{2 j} \leq 1 / t} M(t, j)\left\|u_{0} * \phi_{j}\right\|_{L^{p}}\right)^{p}+\left(\sum_{2^{2 j} \geq 1 / t} M(t, j)\left\|u_{0} * \phi_{j}\right\|_{L^{p}}\right)^{p}=: I_{1}(t)+I_{2}(t) .
\end{aligned}
$$


Here, $\mathcal{F}^{-1}$ is the inverse Fourier transform in $\mathbb{R}^{n}$. By Lemma 4.2 we have $M(t, j) \leq c$ for $t 2^{2 j} \leq 1$. We take $a$ satisfying $-\frac{2}{q}<a<0$ and then use Hölder inequality to get

$$
\begin{gathered}
\int_{0}^{\infty} I_{1}(t)^{\frac{q}{p}} d t \lesssim \int_{0}^{\infty}\left(\sum_{2^{2 j} \leq 1 / t} 2^{-\frac{q}{q-1} a j}\right)^{q-1} \sum_{2^{2 j} \leq 1 / t} 2^{q a j}\left\|\phi_{j} * u_{0}\right\|_{L^{p}}^{q} d t \\
\lesssim \int_{0}^{\infty} t^{\frac{1}{2} q a} \sum_{2^{2 j} \leq 1 / t} 2^{q a j}\left\|\phi_{j} * u_{0}\right\|_{L^{p}}^{q} d t \\
\lesssim \sum_{j=-\infty}^{\infty} 2^{q a j}\left\|\phi_{j} * u_{0}\right\|_{L^{p}}^{q} \int_{0}^{2^{-2 j}} t^{\frac{1}{2} q a} d t=c \sum_{j=-\infty}^{\infty} 2^{-2 j}\left\|\phi_{j} * u_{0}\right\|_{L^{p}}^{q} .
\end{gathered}
$$

Using Lemma 4.2 again, we have $M(t, j) \leq c\left(t 2^{2 j}\right)^{-m} \sum_{0 \leq i \leq n}\left(t 2^{2 j}\right)^{i} \leq c 2^{(2 n-2 m) j} t^{n-m}$ for $t \cdot 2^{2 j} \geq 1$ and $m>0$. We fix $b>0$ and then choose $m$ satisfying $q(n-m)+\frac{1}{2} q b+1<0$, so that we obtain

$$
\begin{gathered}
\int_{0}^{\infty} I_{2}(t)^{\frac{q}{p}} d t \lesssim \int_{0}^{T}\left(\sum_{2^{2 j} \geq 1 / t} 2^{(2 n-2 m) j} t^{n-m}\left\|\phi_{j} * u_{0}\right\|_{L^{p}}\right)^{q} d t \\
\lesssim \int_{0}^{\infty} t^{q(n-m)}\left(\sum_{2^{2 j} \geq 1 / t} 2^{-\frac{q}{q-1} b j}\right)^{q-1} \sum_{2^{2 j} \geq 1 / t} 2^{q b j} 2^{q(2 n-2 m) j}\left\|\phi_{j} * u_{0}\right\|_{L^{p}}^{q} d t \\
\lesssim \int_{0}^{\infty} t^{q(n-m)+\frac{1}{2} q b} \sum_{2^{2 j} \geq 1 / t} 2^{q b j} 2^{q(2 n-2 m) j}\left\|\phi_{j} * u_{0}\right\|_{L^{p}}^{q} d t \\
\lesssim \sum_{j=-\infty}^{\infty} 2^{q b j} 2^{q(2 n-2 m) j}\left\|\phi_{j} * u_{0}\right\|_{L^{p}}^{q} \int_{2^{-2 j}}^{\infty} t^{q(n-m)+\frac{1}{2} q b} d t=c \sum_{j=-\infty}^{\infty} 2^{-2 j}\left\|\phi_{j} * u_{0}\right\|_{L^{p}}^{q} .
\end{gathered}
$$

Therefore, we obtain (4.12). For $k \in \mathbf{N}$ with $k \geq 1$, we have

$$
D_{x}^{k} v(x, t)=\int_{\mathbb{R}^{n}} \Gamma(x-y, t) D_{y}^{k} u_{0}(y) d y .
$$

Using the estimate (4.12), we obtain

$$
\int_{0}^{\infty}\left\|D_{x}^{k} v(\cdot, t)\right\|_{L^{p}\left(\mathbb{R}^{n}\right)}^{q} d t \leq c\left\|D^{k} u_{0}\right\|_{\dot{B}_{p q}^{-\frac{2}{q}\left(\mathbb{R}^{n}\right)}}^{q} \leq c\left\|u_{0}\right\|_{\dot{B}_{p q}^{k-\frac{2}{q}}\left(\mathbb{R}^{n}\right)}^{q} .
$$

Next, we note that

$$
\widehat{D_{t}^{* \frac{1}{2} \alpha}} v(\xi, t)=\sum_{j=-\infty}^{\infty} \hat{\phi}\left(2^{-j} \xi\right)|\xi|^{2} \int_{t}^{\infty} \frac{e^{-s|\xi|^{2}}}{(s-t)^{\frac{1}{2} \alpha}} d s \widehat{u_{0}}(\xi) .
$$

By the same argument, we get

$$
\begin{aligned}
\int_{\mathbb{R}^{n}}\left|D_{t}^{* \frac{1}{2} \alpha} v(x, t)\right|^{p} d x & \leq\left(\sum_{2^{2 j} \leq 1 / t} N(t, j)\left\|u_{0} * \phi_{j}\right\|_{L^{p}}\right)^{p}+\left(\sum_{2^{2 j} \geq 1 / t} N(t, j)\left\|u_{0} * \phi_{j}\right\|_{L^{p}}\right)^{p} \\
& :=J_{1}(t)+J_{2}(t) .
\end{aligned}
$$

Due to Lemma 4.3, we have $N(t, j) \leq c 2^{\frac{1}{2} \alpha j}$ for $t 2^{2 j} \leq 1$. We take $a$ satisfying $-\frac{2}{q}<a<0$ and then use Hölder inequality to get

$$
\int_{0}^{\infty} J_{1}(t)^{\frac{q}{p}} d t \lesssim \int_{0}^{\infty}\left(\sum_{2^{2 j} \leq 1 / t} 2^{-\frac{q}{q-1} a j}\right)^{q-1} \sum_{2^{2 j} \leq 1 / t} 2^{q a j+q \alpha}\left\|\phi_{j} * u_{0}\right\|_{L^{p}}^{q} d t
$$




$$
\begin{gathered}
\lesssim \int_{0}^{\infty} t^{\frac{1}{2} q a} \sum_{2^{2 j} \leq 1 / t} 2^{q a j+q \alpha}\left\|\phi_{j} * u_{0}\right\|_{L^{p}}^{q} d t \\
\lesssim \sum_{j=-\infty}^{\infty} 2^{q a j+q \alpha}\left\|\phi_{j} * u_{0}\right\|_{L^{p}}^{q} \int_{0}^{2^{-2 j}} t^{\frac{1}{2} q a} d t=c \sum_{j=-\infty}^{\infty} 2^{q \alpha-2 j}\left\|\phi_{j} * u_{0}\right\|_{L^{p}}^{q} .
\end{gathered}
$$

Via Lemma 4.3 we note that $N(t, j) \leq c\left(t 2^{2 j}\right)^{-m} \sum_{0 \leq i \leq n}\left(t 2^{2 j}\right)^{i} \leq c 2^{(2 n-2 m) j} t^{n-m}$ for $t \cdot 2^{2 j} \geq 1$ and $m>0$. We fix $b>0$ and then choose $m$ satisfying $q(n-m)+\frac{1}{2} q b+1<0$, so that we obtain

$$
\begin{gathered}
\int_{0}^{\infty} J_{2}(t)^{\frac{q}{p}} d t \lesssim \int_{0}^{\infty}\left(\sum_{2^{2 j} \geq 1 / t} 2^{(2 n-2 m+\alpha) j} t^{n-m}\left\|\phi_{j} * u_{0}\right\|_{L^{p}}\right)^{q} d t \\
\lesssim \int_{0}^{\infty} t^{q(n-m)}\left(\sum_{2^{2 j} \geq 1 / t} 2^{-\frac{q}{q-1} b j}\right)^{q-1} \sum_{2^{2 j} \geq 1 / t} 2^{q b j} 2^{q(2 n-2 m+\alpha) j}\left\|\phi_{j} * u_{0}\right\|_{L^{p}}^{q} d t \\
\lesssim \int_{0}^{\infty} t^{q(n-m)+\frac{1}{2} q b} \sum_{2^{2 j} \geq 1 / t} 2^{q b j} 2^{q(2 n-2 m+\alpha) j}\left\|\phi_{j} * u_{0}\right\|_{L^{p}}^{q} d t \\
\lesssim \sum_{j=-\infty}^{\infty} 2^{q b j} 2^{q(2 n-2 m+\alpha) j}\left\|\phi_{j} * u_{0}\right\|_{L^{p}}^{q} \int_{2^{-2 j}}^{\infty} t^{q(n-m)+\frac{1}{2} q b} d t=c \sum_{j=-\infty}^{\infty} 2^{\alpha j-2 j}\left\|\phi_{j} * u_{0}\right\|_{L^{p}}^{q} .
\end{gathered}
$$

Using the estimate (4.12), we obtain

$$
\int_{0}^{\infty}\left\|D_{t}^{* \frac{1}{2} \alpha} v(x, \cdot)\right\|_{L^{p}\left(\mathbb{R}^{n}\right)}^{q} d x \leq c\left\|u_{0}\right\|_{\dot{B}_{p q}^{\alpha-\frac{2}{q}}\left(\mathbb{R}^{n}\right)}^{q} .
$$

Hence, from (4.14) and (4.15), we complete the proof of Proposition 4.1

Returning to Stokes system (4.1) and (4.2), via the solution formulae (4.5)-(4.5) and the boundedness of $V_{1}, V_{2}, U$ and $E$ (see (4.7)-(4.8) and Proposition 4.1), we have

$$
\|v\|_{\dot{H}_{p q}^{\alpha, \frac{1}{2} \alpha}\left(\mathbb{R}_{+}^{n} \times(0, T)\right)} \leq c\left\|u_{0}\right\|_{\dot{B}_{p q, 0}^{\alpha-\frac{2}{p}}\left(\mathbb{R}_{+}^{n}\right)} .
$$

For the estimate of pressure, since $D: \dot{B}_{p}^{k-\frac{1}{p}}\left(\mathbb{R}^{n-1}\right) \rightarrow \dot{H}_{p}^{k}\left(\mathbb{R}_{+}^{n}\right)$ with $k \geq 0$ is a bounded operator, respectively, we obtain that for $\alpha>1+\frac{1}{p}$,

$$
\begin{gathered}
\int_{0}^{T}\|p(\cdot, t)\|_{\dot{H}_{p}^{\alpha-1}\left(\mathbb{R}_{+}^{n}\right)}^{q} d t=\int_{0}^{T}\left\|D \gamma \partial_{n} E(t) V_{1} u_{0}\right\|_{\dot{H}_{p}^{\alpha-1}\left(\mathbb{R}_{+}^{n}\right)}^{q} d t \\
\leq c \int_{0}^{T}\left\|\partial_{n} E(t) V_{1} u_{0}\right\|_{\dot{H}_{p}^{\alpha-1}\left(\mathbb{R}_{+}^{n}\right)}^{q} d t \\
\leq c \int_{0}^{T}\left\|E(t) V_{1} u_{0}\right\|_{\dot{H}_{p}^{\alpha}\left(\mathbb{R}_{+}^{n}\right)}^{q} d t \leq c\left\|u_{0}\right\|_{\dot{B}_{p q, 0}^{\alpha-\frac{2}{q}}\left(\mathbb{R}_{+}^{n}\right)} .
\end{gathered}
$$

Combining (4.16)-(4.17), we conclude that $(v, p)$ of Stokes system (4.1) and (4.2) satisfies the following estimate:

$$
\|v\|_{\dot{H}_{p q}^{\alpha, \frac{1}{2} \alpha}\left(\mathbb{R}_{+}^{n} \times(0, T)\right)}+\|p\|_{L^{q}\left((0, T) ; \dot{H}_{p}^{\alpha-1}\left(\mathbb{R}_{+}^{n}\right)\right)} \leq c\left\|u_{0}\right\|_{\dot{B}_{p q, 0}^{\alpha-\frac{2}{q}\left(\mathbb{R}_{+}^{n}\right)}} .
$$


As mentioned earlier, following similar procedures as above, we can prove that

$$
\|v\|_{H_{p}^{\alpha, \frac{1}{2} \alpha}\left(\mathbb{R}_{+}^{n} \times(0, T)\right)}+\|p\|_{L^{q}\left((0, T) ; H_{p}^{\alpha-1}\left(\mathbb{R}_{+}^{n}\right)\right)} \leq c\left\|u_{0}\right\|_{B_{p q, 0}^{\alpha-\frac{2}{q}\left(\mathbb{R}_{+}^{n}\right)}} .
$$

Since the proof of (4.19) is on the same track, we skip its details. Summing up above results, the proof of Theorem 1.1 is fulfilled.

Proof of Theorem 1.1 Since the solution of (1.1)-(1.2) is the sum of solutions for (3.1)-(3.2) and (4.1)-(4.2), the proof can be done by combining estimates (3.32)-(3.33) and (4.18)-(4.19) established in section 3 and Section 4. Uniqueness of solution can be established by using duality considerations by following the argument in [28, Theorem 2.1]. More precisely, let $\tilde{v}$ and $\hat{v}$ be two solutions in the sense of distributions (1.13). We set $w:=\tilde{v}-\hat{v}$, which is certainly in $L^{q}\left((0, T) ; L^{p}\left(\mathbb{R}_{+}^{n}\right)\right)$ and we then observe that for any $T^{\prime}<T$ the following equality is satisfied:

$$
\int_{0}^{T^{\prime}} \int_{\mathbb{R}_{+}^{n}} w \cdot\left(-\phi_{t}-\Delta \phi+\nabla \pi\right) d x d t=0
$$

for any compactly supported smooth vector $\phi$ with $\left.\phi\right|_{t=T^{\prime}}=0$ and smooth scalar function $\pi$. Since the vector field $-\phi_{t}-\Delta \phi+\nabla \pi$ is dense in $L^{q^{\prime}}\left((0, T) ; L^{p^{\prime}}\left(\mathbb{R}_{+}^{n}\right)\right)$, it follow that $w=0$. This completes the proof.

\section{Proofs of Theorem 1.2 and Theorem 1.3}

In this section, we present the proofs of Theorem 1.2 and Theorem 1.3 We begin with Theorem 1.2. which is a direct consequence of Theorem 1.1

Proof of Theorem 1.2 In Theorem 1.2, Since $F \in L^{q}\left(0, T ; H_{p, 0}^{\beta}\left(\mathbb{R}_{+}^{n}\right)\right)$, we note that $\nabla \cdot F$ belongs to $L^{q}\left(0, T ; H_{p, 0}^{-1+\beta}\left(\mathbb{R}_{+}^{n}\right)\right.$ and

$$
\|\nabla \cdot F\|_{L^{q}\left(0, T ; H_{p, 0}^{-1+\beta}\left(\mathbb{R}_{+}^{n}\right)\right.} \leq c\|F\|_{L_{q}\left(0, T ; H_{p, 0}^{\beta}\left(\mathbb{R}_{+}^{n}\right)\right)} .
$$

On the other hand, due to the result of Theorem 1.2, we obtain

$$
\begin{gathered}
\|v\|_{H_{p q}^{1+\beta, \frac{1+\beta}{2}}\left(\mathbb{R}_{+}^{n} \times(0, T)\right)} \leq c\|\nabla \cdot F\|_{H_{p q, 0}^{-1+\beta, \frac{-1+\beta}{2}}}\left(\mathbb{R}_{+}^{n} \times(0, T)\right) \\
\|p\|_{L_{q}\left(0, T ; H_{p}^{\beta}\left(\mathbb{R}_{+}^{n}\right)\right)} \leq c\|\nabla \cdot F\|_{L_{q}\left(0, T ; H_{p, 0}^{-1+\beta}\left(\mathbb{R}_{+}^{n}\right)\right)}+c\left\|v_{0}\right\|_{B_{p q, 0}^{1+\beta-\frac{2}{q}}\left(\mathbb{R}_{+}^{n}\right)}, \quad \frac{1}{p}<\beta \leq 1 . \\
\end{gathered}
$$

We observe via the estimate (5.20) that

$$
\|\nabla \cdot F\|_{H_{p q, 0}^{-1+\beta, \frac{-1+\beta}{2}}\left(\mathbb{R}_{+}^{n} \times(0, T)\right)} \leq\|\nabla \cdot F\|_{L^{q}\left(0, T ; H_{p, 0}^{-1+\beta}\left(\mathbb{R}_{+}^{n}\right)\right.} \leq c\|F\|_{L_{q}\left(0, T ; H_{p, 0}^{\beta}\left(\mathbb{R}_{+}^{n}\right)\right)} .
$$

Combining (5.21)-(5.23), the estimates (1.14) and (1.15) are immediate.

We first recall the definition of weak solutions for the Navier-Stokes equations.

Definition 5.1. Let $v_{0} \in L^{2}(\Omega)$ with $\operatorname{div} v_{0}=0$. We say $v$ is a weak solution of (1.19)-(1.20) if $v$ satisfies the following:

(i) $v \in L^{\infty}\left(0, T ; L^{2}(\Omega)\right) \cap L^{2}\left(0, T ; H_{0}^{1}(\Omega)\right)$ and $v$ satisfies

$$
\int_{0}^{T} \int_{\Omega}\left(\frac{\partial \phi}{\partial t}+(u \cdot \nabla) \phi\right) u d x d t+\int_{\Omega} u_{0} \phi(x, 0) d x=\int_{0}^{T} \int_{\Omega} \nabla u: \nabla \phi d x d t
$$

for all $\phi \in \mathcal{C}_{0}^{\infty}(\Omega \times[0, T))$ with $\operatorname{div} \phi=0$. 
(ii) $v$ satisfies divergence free condition, that is, $\int_{\Omega} u \cdot \nabla \psi d x=0$ for any $\psi \in \mathcal{C}^{\infty}(\bar{\Omega})$.

Next, we decompose the nonlinear term of Helmholtz type such that the result of Theorem 1.1 can be applicable.

Lemma 5.2. Suppose that the assumptions in Theorem 1.3 hold. Then the nonlinear term $(v \cdot \nabla) v$ is decomposed as $\operatorname{div}(v \otimes v)=-\nabla \pi+w$, where $w$ has zero divergence and $\pi$ solves in a weak sense

$$
\left\{\begin{array}{cl}
-\Delta \pi=\partial_{x_{i}} \partial_{x_{j}}\left(v_{i} v_{j}\right) & \text { in } \mathbb{R}_{+}^{3}, \\
\pi=0 & \text { on } \partial \mathbb{R}_{+}^{3}
\end{array}\right.
$$

such that the following estimate is satisfied: for almost all $t \in I$

$$
\|\pi(\cdot, t)\|_{\dot{H}_{p}^{\beta}\left(\mathbb{R}_{+}^{3}\right)}+\|w(\cdot, t)\|_{\dot{H}_{p, 0}^{\beta-1}\left(\mathbb{R}_{+}^{3}\right)} \leq C\|(v \otimes v)(\cdot, t)\|_{\dot{H}_{p, 0}^{\beta}\left(\mathbb{R}_{+}^{3}\right)} .
$$

Since standard theories of elliptic equations imply that $\|\pi\|_{\dot{H}_{p}^{\beta}\left(\mathbb{R}_{+}^{3}\right)} \leq C\|(v \otimes v)(\cdot, t)\|_{\dot{H}_{p, 0}^{\beta}\left(\mathbb{R}_{+}^{3}\right)}$ (see also Lemma 3.2) and $w=\nabla \pi+\operatorname{div}(v \otimes v)$, the estimate (5.25) is immediate, and therefore we skip the details of Lemma 5.2. Next lemma shows an estimate of interpolation for fractional derivative of square functions.

Lemma 5.3. Let $0<\beta \leq 1,1 \leq p<\infty$ and $1 / p_{1}+1 / p_{2}=1 / p$ with $1<p_{2}<\infty$. Then, the following a priori estimate holds:

$$
\left\|u^{2}\right\|_{H_{p}^{\beta}\left(\mathbb{R}_{+}^{n}\right)} \leq c\left(\left\|u^{2}\right\|_{L^{p}\left(\mathbb{R}^{n}\right)}+\|u\|_{L^{p_{1}\left(\mathbb{R}_{+}^{n}\right)}}\|u\|_{L^{p_{2}\left(\mathbb{R}_{+}^{n}\right)}}^{1-\beta}\|u\|_{H_{p_{2}}^{1}\left(\mathbb{R}_{+}^{n}\right)}^{\beta}+\|u\|_{L^{2}\left(\mathbb{R}_{+}^{n}\right)}^{2-\beta}\|u\|_{H_{2}^{1}\left(\mathbb{R}_{+}^{n}\right)}^{\beta}\right) .
$$

Proof. We note that

$$
\left\|u^{2}\right\|_{H_{p}^{\beta}\left(\mathbb{R}_{+}^{n}\right)} \approx\left\|u^{2}\right\|_{H_{p}^{\beta}\left(\mathbb{R}^{n}\right)} \approx\left\|u^{2}\right\|_{L^{p}\left(\mathbb{R}^{n}\right)}+\left\|\Delta^{\frac{\beta}{2}} u^{2}\right\|_{L^{p}\left(\mathbb{R}^{n}\right)}
$$

where

$$
\Delta^{\frac{\beta}{2}} f(x)=-c(n, \beta) \int_{\mathbb{R}^{n}} \frac{f(x)-f(y)}{|x-y|^{n+\beta}} d y
$$

with a positive constant $c(n, \beta)$. Simple computations show that

$$
\Delta^{\frac{\beta}{2}} u^{2}(x)=2 u(x) \Delta^{\frac{\beta}{2}} u(x)+c(n, \alpha) \int_{\mathbb{R}^{n}} \frac{(u(x)+u(y))^{2}}{|x-y|^{n+\beta}} d y .
$$

Hence, we obtain

$$
\left\|u \Delta^{\frac{\beta}{2}} u\right\|_{L^{p}} \leq\|u\|_{L^{p_{1}}}\left\|\Delta^{\frac{\beta}{2}} u\right\|_{L^{p_{2}}} \leq\|u\|_{L^{p_{1}}}\|u\|_{H_{p_{2}}^{\beta}} .
$$

We recall that for $1 \leq r<\infty$ (see e.g. [2, Theorem 6.4.4])

$$
\|u\|_{H_{r}^{\beta}\left(\mathbb{R}^{n}\right)} \leq \begin{cases}\|u\|_{B_{r}^{\beta}\left(\mathbb{R}^{n}\right)}, & 1 \leq r \leq 2, \\ \|u\|_{B_{r 2}^{\beta}\left(\mathbb{R}^{n}\right)}, & 2 \leq r<\infty .\end{cases}
$$

Via interpolations $\left(L^{r}\left(\mathbb{R}^{n}\right), H_{r}^{1}\left(\mathbb{R}^{n}\right)\right)_{\beta}=B_{r}^{\beta}\left(\mathbb{R}^{n}\right)$ and $\left(L^{r}\left(\mathbb{R}^{n}\right), H_{r}^{1}\left(\mathbb{R}^{n}\right)\right)_{\beta, 2}=B_{r 2}^{\beta}\left(\mathbb{R}^{n}\right)$ (refer to 2 , section 3.5]), we have $\|u\|_{H_{p_{2}}^{\beta}\left(\mathbb{R}^{n}\right)} \leq c\|u\|_{L^{p_{2}\left(\mathbb{R}_{+}^{n}\right)}}^{1-\beta}\|u\|_{H_{p_{2}}^{1}\left(\mathbb{R}_{+}^{n}\right)}^{\beta}$ and therefore,

$$
\left\|u \Delta^{\frac{\beta}{2}} u\right\|_{L^{p}\left(\mathbb{R}^{n}\right)} \leq c\|u\|_{L^{p_{1}}\left(\mathbb{R}_{+}^{n}\right)}\|u\|_{L^{p_{2}\left(\mathbb{R}_{+}^{n}\right.}}^{1-\beta}\|u\|_{H_{p_{2}}^{1}\left(\mathbb{R}_{+}^{n}\right)}^{\beta} .
$$


Using similar arguments for the second term in (5.28), we get

$$
\begin{gathered}
\left(\int_{\mathbb{R}^{n}}\left(\int_{\mathbb{R}^{n}} \frac{|u(x)-u(y)|^{2}}{|x-y|^{n+\beta}} d y\right)^{p} d x\right)^{\frac{1}{p}}=\left(\int_{\mathbb{R}^{n}}\left(\int_{\mathbb{R}^{n}} \frac{|u(x)-u(y)|^{2}}{|x-y|^{n+2 \frac{\beta}{2}}} d y\right)^{\frac{2 p}{2}} d x\right)^{\frac{2}{2 p}} \\
\left.\leq\|u\|_{B_{2,2 p}^{\frac{\beta}{2}}}^{2} \leq c \mathbb{R}^{n}\right) \\
\leq c u\left\|_{L^{2}\left(\mathbb{R}_{+}^{n}\right)}^{2-\beta}\right\| u \|_{H_{2}^{1}\left(\mathbb{R}_{+}^{n}\right) .}^{\beta} .
\end{gathered}
$$

From (5.27), (5.29) and (5.30), we deduce the lemma.

Next we give the proof of Theorem 1.3 .

Proof of Theorem 1.3 Let $\hat{p}=p-\pi$, where $\pi$ is given in (5.24). We then obtain by the result of Theorem 1.1 that

$$
\|v\|_{L^{q}\left(0, T ; H_{p}^{1+\beta}\left(\mathbb{R}_{+}^{3}\right)\right)}+\|\hat{p}\|_{L^{q}\left(0, T ; H_{p}^{\beta}\left(\mathbb{R}_{+}^{3}\right)\right)} \leq c\|w\|_{L^{q}\left(0, T ; H_{p, 0}^{\beta-1}\left(\mathbb{R}_{+}^{3}\right)\right)}+c\left\|v_{0}\right\|_{B_{p q, 0}^{1+\beta-\frac{2}{q}}\left(\mathbb{R}_{+}^{3}\right)},
$$

where $w$ is given in Lemma 5.2 Using the estimate (5.25) after integrating it in time variable, we have (1.24). It remains to show that the righthand side in (1.24) is bounded. Due to the estimate (5.26) by choosing $p_{2}=2$, we note that

$$
\left\|v^{2}\right\|_{H_{p, 0}^{\beta}\left(\mathbb{R}_{+}^{3}\right)} \leq C\left(\left\|v^{2}\right\|_{L^{p}\left(\mathbb{R}_{+}^{3}\right)}+\|v\|_{L^{2}\left(\mathbb{R}_{+}^{3}\right)}^{2-\beta}\|v\|_{H_{2}^{1}\left(\mathbb{R}_{+}^{3}\right)}^{\beta}+\|v\|_{L^{p_{1}\left(\mathbb{R}_{+}^{3}\right)}}\|v\|_{L^{2}\left(\mathbb{R}_{+}^{3}\right)}^{1-\beta}\|v\|_{H_{2}^{1}\left(\mathbb{R}_{+}^{3}\right)}^{\beta}\right)
$$

where $1 / p_{1}=1 / p-1 / 2$. Taking $L^{q}$-norm in time variable, we obtain

$$
\begin{gathered}
\left\|v^{2}\right\|_{L^{q}\left(0, T ; H_{p, 0}^{\beta}\left(\mathbb{R}_{+}^{3}\right)\right)} \leq C\|v\|_{L^{2 q}\left(0, T ; L^{2 p}\left(\mathbb{R}_{+}^{3}\right)\right)}^{2}+\|v\|_{L^{\infty}\left(0, T ; L^{2}\left(\mathbb{R}_{+}^{3}\right)\right)}^{2-\beta}\|v\|_{L^{2}\left(0, T ; H_{2}^{1}\left(\mathbb{R}_{+}^{3}\right)\right)}^{\beta} \\
+C\|v\|_{L^{\frac{2 q}{2-q \beta}}\left(0, T ; L^{\left.p_{1}\left(\mathbb{R}_{+}^{3}\right)\right)}\right.}\|v\|_{L^{\infty}\left(0, T ; L^{2}\left(\mathbb{R}_{+}^{3}\right)\right)}^{1-\beta}\|v\|_{L^{2}\left(0, T ; H_{2}^{1}\left(\mathbb{R}_{+}^{3}\right)\right)}^{\beta} .
\end{gathered}
$$

Since $2<p_{1} \leq 6$ and $3 / p_{1}+(2-q \beta) / q=3 / 2$, the last term in (5.31) is finite. This completes the proof.

\section{Acknowledgements}

T.-K. Chang's work was partially supported by NRF-2009-0088692 and K. Kang's work was partially supported by NRF-2012R1A1A2001373.

\section{References}

[1] C. Bennett \& R. Sharpley, Interpolation of Operators, Academic press, Inc. 1988.

[2] J. Bergh and J. Lofstrom, Interpolation Spaces, An Introduction, Springer-Verlag, Berlin 1976.

[3] L. Caffarelli, R. Kohn \& L. Nirenberg, Partial regularity of suitable weak solutions of the NavierStokes equations, Comm. Pure Appl. Math. 35, (1982), 771-831.

[4] H. Choe \& J. L. Lewis, On the singular set in the Navier-Stokes equations, J. Funct. Anal. 175, (2000), no. 2, 348-369.

[5] L. Escauriaza, G. Seregin \& V. Šverák, $L^{3, \infty}$-solutions of Navier-Stokes equations and backward uniqueness, (Russian) Uspekhi Mat. Nauk 58, (2003), no. 2(350), 3-44; translation in Russian Math. Surveys 58, (2003), no. 2, 211-250. 
[6] E. B. Fabes, B. F. Jones \& N. M. Riviére, The initial value problem for the Navier-Stokes equations with data in $L^{p}$, Arch. Rational Mech. Anal. 45, (1972), 222-240.

[7] M. Giga, Y. Giga \& H. Sohr, $L^{p}$ estimates for the Stokes system, Lect. Notes Math. 1540, (1993), 55-67.

[8] Y. Giga, Solutions for semilinear parabolic equations in $L^{p}$ and regularity of weak solutions of the Navier-Stokes system, J. Differential Equations 62, (1986), no. 2, 186-212.

[9] Y. Giga \& H. Sohr, Abstract $L^{p}$ estimates for the Cauchy problem with applications to the Navier-Stokes equations in exterior domains, J. Funct. Anal. 102, (1991), 72-94.

[10] S. Gustafson, K. Kang \& T. Tsai, Regularity criteria for suitable weak solutions of the NavierStokes equations near the boundary, J. Differential Equations, 226 (2006), no. 2, 594-618.

[11] S. Gustafson, K. Kang \& T. Tsai, Interior regularity criteria for suitable weak solutions of the Navier-Stokes equations, Comm. Math. Phys., 273 (2007), no. 1, 161-176.

[12] E. Hopf, Über die Anfangswertaufgabe für die hydrodynamischen Grundgleichungen (German), Math. Nachr., 4 (1951), 213-231.

[13] B. Jawerth, The trace of Sobolev and Besov spaces if $0<p<1$, Studia Mathmetica, 62, no 1, (1978), 65-71.

[14] D. Jerison \& C. Kenig, The inhomogeneous Dirichlet Problem in Lipschitz domains, J. of Funct. Anal, 130, (1995), 161-219.

[15] A. Jonsson \& H. Wallin, A whitney extension theorem in $L_{p}$ and besov spaces, Ann.Inst. Fourier Grenoble, 28, (1978), 139-192.

[16] K. Kang, On boundary regularity of the Navier-Stokes equations, Comm. Partial. Differential Equations, 29, no 7-8, (2004), 955-987.

[17] H. Koch \& V. A. Solonnikov, $L_{p}$-estimates of the first-order derivatives of solutions to the nonstationary stokes problem, Nonlinear Problems in Mathematical Physics and Related Topics I, Springer International Mathematical Series Volume 1, (2002), 203-218.

[18] O. A. Ladyzenskaja, Uniqueness and smoothness of generalized solutions of Navier-Stokes equations, (Russian) Zap. Naučn. Sem. Leningrad. Otdel. Mat. Inst. Steklov. (LOMI) 5, (1967), $169-185$.

[19] J. Leray, Sur le mouvement d'un liquide visqueux emplissant l'espace (French), Acta Math., 63 (1934), no. 1, 193-248.

[20] P. I. Lizorkin, Multipliers of Fourier integrals and estimates of convolutions in spaces with mixed norm, Applications. (Russian) Izv. Akad. Nauk SSSR Ser. Mat. 34, (1970), 218-247.

[21] P. Maremonti and V. A. Solonnlkov, Estimates for solutions of the nonstationary stokes problem in anisotropic Sobolev spaces with mixed norm, Journal of Mathematical Sciences, 87, no. 5, (1997), 3859-3877.

[22] G. Prodi, Un teorema di unicita per le equazioni di Navier-Stokes, (Italian) Ann. Mat. Pura Appl. 48, (1959), no. 4, 173-182.

[23] V. Scheffer, Hausdorff measure and the Navier-Stokes equations, Comm. Math. Phys. 55, (1977), $97-112$. 
[24] V. Scheffer, Boundary regularity for the Navier-Stokes equations in a half-space, Comm. Math. Phys. 85, (1982), no. 2, 275-299.

[25] G. A. Seregin, Local regularity of suitable weak solutions to the Navier-Stokes equations near the boundary, J. Math. Fluid Mech. 4, no. 1, (2002), 1-29.

[26] J. Serrin, On the interior regularity of weak solutions of the Navier-Stokes equations, Arch. Rational Mech. Anal. 9, (1962), 187-195.

[27] V. Solonnikov, Estimates for solutions of nonstationary Navier-Stokes equations, (Russian) Boundary value problems of mathematical physics and related questions in the theory of functions, 7. Zap. Naucn. Sem. Leningrad. Otdel. Mat. Inst. Steklov. (LoMI) 38: p.153-231(1973).; translated in J. Soviet Math., 8, (1977), 467-529.

[28] A. V. Solonnikov, Estimates for solutions of the nonstationary Stokes problem in anisotropic Sobolev spaces and estimates for the resolvent of the Stokes operator, (Russian) Uspekhi Mat. Nauk, 58, no. 2(350), 123-156 (2003); translation in Russian Math. Surveys 58, (2003), no. 2, $331-365$.

[29] E. Stein, Singular integrals and differentiability properties of functions, Princeton University Press, 1970.

[30] M. Struwe, On partial regularity results for the Navier-Stokes equations, Comm. Pure Appl. Math. 41, (1988), 437-458.

[31] H. Triebel, Interpolation Theory, Function Space, Differential Operators, Noth-Holland Pubilshing Company, 1978.

[32] S. Ukai, A solution formula for the Stokes equation in $\mathbb{R}_{+}^{n}$, Comm. Pure Appl. Math. 40, no. $5,(1987), 611-621$.

Tongkeun Chang

Department of Mathematics

Yonsei University

Seoul, Republic of Korea

chang7357@yonsei.ac.kr
Kyungkeun Kang

Department of Mathematics

Yonsei University

Seoul, Republic of Korea

kkang@yonsei.ac.kr 